\title{
Percepción de innovación en las organizaciones. Percepción de innovación en organizaciones de España y Latinoamérica
}

\section{Perception of innovation in organizations. Perception of innovation in organizations of Spain and Latin América}

\author{
Silvia da Costa** \\ Universidad del País Vasco/Euskal Herriko Universitatea, España \\ Darío Páez ${ }^{* * *}$ \\ Universidad del País Vasco/Euskal Herriko Universitatea, España \\ Sonia Gondim**** \\ Universidad Federal de Bahía, Brasil \\ Marta Rodríguez ${ }^{* * * * *}$ \\ Universidad del País Vasco/Euskal Herriko Universitatea, España \\ Silvia Mazzieri******* \\ Universidad Tecnológica de Trenque Lauquen, Argentina

Alejandro Torres
Universidad de Defensa Nacional, Argentina
Gaudipersonas
G*******
Institución del Autor, Chile
Flor Sánchez
*********
Universidad Autónoma de Madrid, España

Patricia Jiménez

Universidad Autónoma de Ciudad Juárez, México

** Depto. Psicología Social y Metodología de las Ciencias del Comportamiento. Candidata Predoctoral. Correspondencia: Silvia da Costa, Departamento de Psicología Social y Metodología de las Ciencias del Comportamiento, Avenida de Tolosa 70, Donostia/San Sebastián. Correo electrónico: silviacristina.dacosta@ehu.eus

***Catedrático Psicología social. Correo electrónico: dario.paez@ehu.eus

*****Professor. Doctor of Psychology. Correo electrónico: sggondim@gmail.com

*****Professor. Doctor of Psychology. Correo electrónico: marta.rodriguez@ehu.eus

******PhD. In Psichology. Correo electrónico: silvia_mazzieri@yahoo.com.ar

********Coronel Mayor, asesor del área de Ciencia y Técnica de la Universidad de la Defensa Nacional. Correo electrónico: aletorres40@yahoo.com.ar

*********Gaudipersonas. Consultoría y Selección, Santiago de Chile, Chile. Correo electrónico: mpais@gaudi.cl

**********Flor Sánchez. Professor. Doctor of Psychology. Correo electrónico: flor.sanchez@uam.es

\section{RESUMEN}

Se indaga sobre la percepción de factores psicosociales de la innovación en organizaciones de España y Latinoamérica utilizando una medida construida a partir de la evidencia meta-analítica. El inventario de conductas y actitudes colectivas favorables a la innovación muestra adecuada fiabilidad y validez predictiva, de criterio, convergente y discriminante. Se percibe que las características de rol laboral, el liderazgo, los factores grupales y de clima emocional se dan en forma moderada y en menor medida, los factores organizacionales. La importancia atribuida a la innovación y su valoración es percibida como superior a su presencia y en mayor medida en otras organizaciones que en la propia. La percepción global de innovación se asoció positiva y significativamente a los factores psicosociales mostrando relevancia las características de rol laboral, el factor organizacional y, en menor medida el liderazgo. Este es mediador entre los factores organizacionales, de rol y la innovación. Congruentemente con los meta-análisis la asociación fue menos relevante con los factores individuales, grupales y de clima socio- emocional. Se discuten resultados e implicaciones prácticas.

Palabras clave

creatividad aplicada, innovación, factores psicosociales, medición, organizaciones. 


\begin{abstract}
This study examines the perception of factors of innovation in samples of experts and workers from Spain and LatinAmerica, using a scale based on meta-analytic evidence. The scale of collective behavior and attitudes favorable to innovation shows satisfactory reliability and criterion validity. Participants perceive that role, group, leadership, climate and psychological factors of innovations exist at a moderate level, while perceive that organizational structure of innovation are present at a lower extent. Valuation of innovation is perceived as higher than actual degree of it, and both valuation and frequency of innovation are perceived as higher in others organizations than in the organization to which participants belongs. Perceived level of innovation was strongly associated to organizational and role factors, and in a lower extent to leadership factors, while individual and group factors were less relevant, congruently with metaanalysis. Positive and innovative leadership play a meditational rol between organizational and role factors and innovation. Implications of results for organizations are discussed.
\end{abstract}

Keywords

applied creativity, innovation, measurement scale, psychosocial factors, organizations.

\section{Interés en la creatividad e innovación organizacional}

Además de la investigación académica (p.e., Csikszentmihalyi, 2011; Scott, 2008; West \& Farr, 2009) la creatividad (Garaigordobil, 2003; Hennesey \& Amabile, 2010) y la innovación (Adamsky, 2010; Anderson, Potocnik, \& Zhou, 2014; Foley, 2012; ordan, 2015; Landström \& Lohke, 2010; Patterson, Kerrin, \& Gatto- Roissard, 2009) son leit motiv de instituciones internacionales (Cachia, Ferrari, Ala-Mutka,

\& Punie, 2010; Banco Interamericano de Desarrollo, 2011; European Commission, 2016; European Union, 2009; Navarro \& Olivares, 2016; Unesco, 2016), nacionales (Aboal et al., 2015; Botín, 2012; Jiménez \& Bruzzo, 2016; Mincyt, 2015; Mincyt, 2016-2019; Mineco, 2013; Plan Uruguay, 2015; SIICyT, 2013) y territoriales (Confederación empresarial de Madrid-CEOE, 2015; Generalitat de Catalunya,

2012; Hezkuntza, 2012, Innobasque, 2016; Sánchez Bueno, 2008). Dicho interés genera un discurso compartido sobre su centralidad e importancia para adaptarse a los desafíos educacionales, económicos, militares y sociales del mundo actual (p.e., Fundación Botín,2014; Bureau of European Policy Advises, 2011; Cameron \& Spreitzer, 2012; Crespi, Navarro, \& Zuñiga, 2010; Dutta, Lanvin, \& Wunsch-Vincent, 2015; EC, 2016; Fagerberg, Mowery, \& Richard, 2009; Horowitz, 2010; Naciones Unidas/PNUD/Unesco, 2014; Riivari, Lämsä, Kujala, \& Heiskanen, 2012). También se indica que existe evidencia sobre la innovación como mejora de las organizaciones y su desempeño facilitando el rendimiento y asegurando su supervivencia en un mundo gobalizado, competitivo y complejo (Crespi \& Zuñiga, 2010; Bowen, Rostami, \& Steel, 2010; European Union, 2016; Mumford, Hester, \& Robledo, 2012; OECD, 2005; Robbins \& Judge, 2016; Romero, 2014).

\section{La innovación como transformación, crecimiento y competitividad a nivel macro- social y en las organizaciones}

La innovación se plantea como requisito para la sobrevivencia de las organizaciones (Larracoechea, 2013; Scarpinelli, 2013) en el marco de un capitalismo concebido como de lucha a muerte entre gladiadores (Moscovici, 1976; Schumacher \& Wasieleski, 2013), algo así como una visión darwiniana social a lo Spencer ${ }^{[1]}$ o donde solo sobreviven los más fuertes (Cabrera, Tacoronte, \& Medina, 2008; Robbins \& Judge, 2016). Sin embargo, y a pesar del discurso glorificador en el que la relación entre la innovación y el desarrollo económico se postula como una verdad macro económica indudable, las revisiones narrativas describen la evidencia como mixta, no concluyente o contradictoria (Rosenbusch, Brinckmann, \& Bausch, 2011).

La evidencia - limitada - revisada en dos metaanálisis sugiere que la innovación predice ligeramente una mejora del rendimiento económico $(\mathrm{r}=0.14 \mathrm{y} \mathrm{r}=$ $0.16)$ y con mayor intensidad se asocia a una mejora del rendimiento organizacional $(r=0.40)$. (Bowen et al., 2010; Rosenbusch et al., 2011). Por ejemplo, un aspecto que destaca en la innovación es el del emprendimiento (González \& Peña, 2007) ignorando su alta tasa de fracaso cercana al $80 \%$ (Song, Podoynitsyna, van Der Bij, \& Halman, 2008; Rauch \& Frese, 2007; Watson \& Everett, 1996) y su vinculación con formas distorsionadas del pensamiento social (Cope, 2011). Se ha confirmado que los sesgos de cognición social (Fiedler \& von Sydow, 2015; 
Gigerenzer \& Gaissmaier, 2011; Kahneman, 2012; De Neys, 2010; von Sydow \& Braus, 2016) en particular el de sobreconfianza se asocian con el emprendimiento (Zhao, Scott, \& Lumpkin, 2010). Camerer y Lovallo (1999) mostraron experimentalmente que este sesgo se encuentra relacionado con los elevados fracasos a la hora de emprender nuevos negocios (Cooper, Dunkelberg, \& Woo, 1988; INE, 2014; Song et al., 2008), aunque existen otras variables de influencia a tener en cuenta (ver p.e., Caprile, 2012; EC, 2017; Injuve, 2015 ; Ma, 2009; Nonaka \& Toyama, 2005; Villamizar, 2012).

El complejo panorama actual es visto por investigadores como oportunidades y desafíos a la sociedad y a la estructura productiva de los países a nivel mundial y organizacional (Mumford et al., 2012; Robbins \& Judge, 2016). Parece existir suficiente evidencia acerca de que la creatividad y la innovación son fundamentales para el crecimiento de las organizaciones. Sin embargo, ha de señalarse críticamente, que la glorificación — sobre todo de la innovación- da por supuesto variables y acontecimientos (p. e., Ashkanasy \& Härtel, 2014; Benito, Platero, \& Rodríguez, 2012; Cañibano \& Castro, 2010; Cotec, 2016; Davis- Blake \& Broschak, 2009; Faleg, 2016; Gondim et al., 2015; González, Navarro, \& Peña, 2010; Pelkmans \& Renda, 2014; Pisano, Lange, \& Berger, 2015; Sarooghi, Libaers, \& Burkemper, 2015; Yesil \& Sozbilir, 2013) que influencian la realidad social, organizacional-empresarial e institucional (Scott, 2008). Dicha glorificación podría contribuir a sobreestimar la percepción que tienen las personas sobre la innovación en sus organizaciones dándose una suerte de incongruencia entre lo que se espera y lo que hay (Barsade, Barsade, \& Licina, 2012; Gondim et al., 2015; Noe, Clarke, \& Klein, 2014; Robbins \& Judge, 2016; European Union, 2015). Un estudio con profesores-maestros y profesionales de la intervención social en Chile, Euskadi-País Vasco y Uruguay encontró que el bajo apoyo social y liderazgo de calidad, el alto nivel de estrés (Istas, 2010) y la alta expresión de emociones negativas en el rol (Ortiz, Navarro, García, Rames, \& Manassero, 2012) destacaban entre otras variables analizadas, aunque un rol laboral complejo y autónomo así como el mayor apoyo y liderazgo de calidad reforzaban la innovación y el bienestar (da Costa, Páez, Hermosilla et al., 2016; Hervás \& Vázquez, 2013). El aumento $\mathrm{o} \mathrm{su}$ percepción- de la carga de trabajo, la tensión de resolver problemas complejos, la incertidumbre sobre sus resultados y la falta de claridad de rol (p.e., cuestionar la relación con los pares y superiores porque se perturban los patrones rutinarios, provocar inseguridad y resistencia al cambio en personas del grupo de trabajo afectando la relación entre ellas, los enfoques contradictorios sobre la resolución de problemas y la competencia entre las personas) son costes de la innovación (da Costa, Páez, \& Sánchez, 2016; Hermosilla, Amutio, da Costa, \& Páez, 2016; Huhtala \& Parzefall, 2007). Otro estudio encontró que si bien la innovación puede ser costosa para el bienestar subjetivo y psicológico (Miller, Mark, \& Michaelson, 2008) la mayor percepción de inteligencia emocional (IE) y de un clima socioemocional positivo en su organización se asociaba al bienestar vinculado a la calidad de vida y la salud (da Costa, Páez et al., 2016; Vilagut et al., 2005) - al menos en algunas de las muestras analizadas - En este sentido, la internalización de la innovación como recurso para el bienestar personal, la alta inteligencia y creatividad emocional (Huhtala \& Parzefall, 2007; O’Boyle, Humphrey, Pollack, Hawver, \& Story, 2011; Oriol, Amutio, Mendoza, da Costa, \& Miranda, 2016) así como un uso adaptativo de estrategias de interregulación en uno mismo y en los otros (Belbin, 2011; da Costa, Páez, Oriol, \& Unzueta, 2014; Gross, 2015; Peña-Sarrionandia, Mikolajczak, \& Gross, 2015) pueden contribuir a disminuir los síntomas de malestar y facilitar la innovación (Csikszentmihalyi, 2003; Gardner \& Csikszentmihalyi, 2002; Huhtala, Kangas, Lämsä, \& Feldt, 2013).

En síntesis, en el impulso de la innovación y para estimular el cambio, las organizaciones se enfrentan a desafíos tales como aumentar o mantener la flexibilidad, la integración, los recursos y la productividad: mejorar la calidad y enfrentarse a los competidores con un flujo continuo de bienes y servicios innovadores: reforzar la experimentación, estimular la creatividad del personal así como reconocer y aceptar los fracasos inherentes a la innovación. Además, es preciso gestionar la temporalidad, los diferentes valores culturales y lo impredecible en un mundo laboral cambiante. Finalmente, crear una cultura organizacional que se beneficie del cambio: motivar, dirigir y tomar decisiones de colaboración online, así como buscar equilibrar el trabajo y las responsabilidades familiares 
son otros aspectos importantes (Buffet, Gervais, Liddle, \& Eechelaert, 2013; Robbins \& Judge, 2016; Vera Calzaretta, Carrasco-Dajer, da Costa, \& Páez, 2015).

\section{Un instrumento de diagnóstico}

Robbins y Judge (2016) plantean que "nunca había sido tan importante para los gerentes entender el comportamiento organizacional como lo es actualmente". Las personas entran a su organización con una personalidad establecida, valores y actitudes relativamente arraigadas y competencias (a veces son o conocimientos, o capacidades y/o habilidades) que influyen en el comportamiento. La importancia de tener en cuenta su percepción se justifica en que la interpretación del ambiente de trabajo va a influir en el nivel de motivación, los aprendizajes y el comportamiento individual (Robbins, 2008).

La evidencia sintetizada en los meta-análisis indica cuáles son los factores psicosociales importantes para la explicación de la creatividad aplicada o innovación organizacional (Hammond, Neff, Farr, Schwall, \& Zhao, 2011; Hülsheger, Anderson, \& Salgado, 2009; Hunter, Bedell, \& Mumford, 2007; Ma, 2009): las características organizacionales y del rol laboral se asocian fuertemente a la creatividad aplicada o innovación, mientras que los procesos grupales y de liderazgo lo hacen con menor fuerza, y los factores individuales lo hacen más débilmente (da Costa, Páez, Sánchez, Garaigordobil, \& Gondim, 2015; da Costa, Páez, Sánchez, Gondim, \& Rodríguez, 2014). La construcción de una escala de medida que integra ambos constructos se justifica por la interrelación entre creatividad e innovación que permite considerarlos procesos complementarios en las organizaciones (Binnewies \& Gromer, 2012; Gondim et al., 2015).

Los objetivos de este estudio han sido: 1. Destacar la importancia de la creatividad e innovación organizacional así como los desafíos — más que las dificultades- que genera la innovación como motor de transformación, crecimiento y competitividad en las organizaciones; 2. Presentar un instrumento de diagnóstico (F.I.N.O., da Costa, Páez, Sánchez et al., 2014) e indagar en qué medida las personas trabajadoras encuestadas perciben que los factores psicosociales de la innovación se dan en sus organizaciones. Finalmente, se quiere conocer la estructura interna del inventario en la que dichos factores se describen bajo la forma de conductas y actitudes observables en el ámbito laboral (ver Tabla 2). Se espera que: a) los factores citados se asocien a un indicador global de innovación (modelo 1); b) muestren mayor fuerza los factores organizacionales y de rol laboral que la percepción de liderazgo, los factores grupales y los psicológicos individuales, como indican los meta-análisis. Se postula que será el factor de liderazgo que mide el apoyo a la innovación de las y los directivos, quien medie entre las características de rol, el factor organizacional y la percepción global de innovación (modelo 2). Para finalizar, en este estudio se analiza si el liderazgo y el factor organizacional que mide integración entre grupos y oferta de recursos, son moderadores de las relaciones entre las características de rol laboral y la percepción global de innovación justificado por la utilidad de aumentar los beneficios del buen diseño del trabajo (ver modelo 3 ).

\section{Método \\ Participantes}

El estudio se llevó a cabo con una muestra total de 900[2] personas, $52 \%$ hombres entre 18 y 65 años $(\mathrm{M}=31.74$, DT $=10.64)$. Si bien no se realizó en esta fase muestreo aleatorio estratificado, si se condicionó la participación a) tener una relación contractual con la organización sobre la que se respondía en el momento de aplicar la encuesta, b) ser capaz de comprender lo que se estaba preguntando, c) hacerlo en castellano, euskera, o portugués. De las personas participantes $n=16$ pertenecían a una muestra inicial (piloto expertos) de Chile y País Vasco (63\% hombres Medad = 42.20, DT $=11.55), \mathrm{n}=50$ a una provincia de Buenos Aires, Argentina (66\% hombres, Medad = 29.92, DT = 7.39); $\mathrm{n}=340$ a la muestra de Brasil, $94 \%$ residentes en ese país $(59 \%$ mujeres, Medad $=36.32, \mathrm{DT}=10.45) ; \mathrm{n}=268$ a organizaciones de tres comunidades autónomas de España (n(166) de un centro de educación superior público), 54\% hombres, edad (Medad2muestras $=43.59$, DT $=$ 10.91) 3 muestras $=71 \%$ entre 30 y 67 años); $n=133$ a organizaciones de un municipio de México (60\% hombres, Medad $=32.32$, DT $=8.28)$ y $\mathrm{n}=315 \mathrm{a}$ cadetes militares de $3^{\circ}$ y $4^{\circ}$ año de un país de América Latina, pertenecientes a distintas armas o 
especialidades $(88 \%$ varones, Medad $=23.52, \mathrm{DT}=$ 2.39). La encuesta original se realizó en castellano, la traducción fue realizada por las investigadoras bilingües del estudio y cuando fue necesario, con la colaboración de expertas/os.

\section{Instrumentos y procedimiento}

Datos sociodemográficos. Se indagó en algunos de los datos que los estudios conciben relevantes para la innovación o creatividad aplicada (da Costa et al., 2015; EC, 2016) (ver Tabla 1). El sexo era punto biserial $1=$ hombre, $2=$ mujer. La edad se codificó en grupos de 5 años hasta los 30 años y de los 30 a los 50 en grupos de 10 años, siendo el 1 $=20$ a 25 años, el $5=50$ a 67 años y el $6=+$ de 67 años. El nivel educativo se dicotomizó en $0=$ hasta educación terciaria, $1=$ nivel educativo terciario $(76 \%)$. ¿Ud. ocupa algún puesto gerencial? se respondía con una variable dicotómica $0=$ no (77\%) $1=$ si. La variable sector al que pertenece la organización se codificó con los valores $1=$ público $(56 \%), 2=$ privado $(41 \%), 3=$ tercer sector (ONGs...), la variable empresa familiar con los valores $0=$ no $(88 \%), 1=$ si. Respecto a la variable tamaño de la organización (cantidad de empleados) se establecieron los intervalos: $1=$ hasta $10(6.6 \%)$, $2=11$ a $50(14.4 \%), 3=51$ a $100(5.4 \%), 4=101$ a $500(14.5 \%), 5=$ más de $500(59 \%)$. En el caso de las empresas, también se formuló la pregunta: ¿Esta organización tiene participación de capital extranjero? asignando los valores: $0=$ no $(81 \%), 1=$ si.

\section{Factores de innovación en las organizaciones} (F.I.N.O). (da Costa, Páez, Sánchez, et al., 2014). El inventario fue creado como instrumento de diagnóstico para ser respondido por personas con vinculación contractual a ellas. Consta de dos preguntas abiertas [3] (ver análisis preliminares en Gondim et al., 2015) que miden la conceptualización de los constructos sobre los que se indaga; mide también la percepción de los factores psicosociales de innovación en su organización [4] por medio de reactivos o ítems y una pregunta de distanciamiento de la experiencia general y personal (ver Tabla 2). Se responde con una escala tipo Likert con valores que oscilan entre $1=$ nada aplicable y $7=$ muy aplicable. La escala se completa con un apartado de equipos - preguntas
22 a 37 [5] - . En este documento solo se analizan las cuestiones que conforman los factores explicitados en la Tabla 2.

Percepción global de innovación. Se calcula sumando el ítem 38b (en su organización, el grado de innovación es) con el 38d (en su organización la valoración o importancia atribuida a la innovación es).

El estudio se desarrollo entre los años 2013 (ver da Costa et al., 2015; da Costa, Páez, Sánchez, et al., 2014) y 2016 en distintos proyectos en los que además del inventario F.I.N.O se respondieron escalas de desarrollo profesional, calidad de vida y síntomas de estrés, liderazgo y afectividad (ver p.e, da Costa, Páez et al., 2016; da Costa, Páez, Hermosilla et al.,2016). El F.I.N.O se desarrolló en versión papel para aplicación in situ y on line. Se solicitó consentimiento informado y se garantizó la confidencialidad de la información. La obtención de la muestra se realizó en diferentes etapas. Primero se recogió la percepción de expertos considerados clave en organizaciones de perfil innovador en el País Vasco y Chile (la tarea fue realizada por dos autores de la escala y una consultora con amplia experiencia en creatividad e innovación). La muestra de Brasil estuvo a cargo de otra de las autoras de la escala y fue recogida en dos fases a través del link de su grupo de investigación en ese país $(30 \%$ de la muestra total, $8 \%$ respondió en castellano). La recolección de datos en un municipio de Argentina se realizó mediante convenio de colaboración con un Centro de Educación Superior Tecnológico y estuvo a cargo de una investigadora responsable y su equipo ( $4.5 \%$ de la muestra). Dos autores de la escala pasaron in situ la encuesta en el Centro de Educación Superior Militar con el apoyo de un equipo de investigación de ese país y los superiores en colaboración con el proyecto (cadetes con formación basada en competencias para la toma de decisiones fueron el $28 \%$ de la muestra total). En una segunda etapa con la gestión de una de las autoras de la escala y el seguimiento de otra, se recogieron datos en un Centro público de Educación Superior del País Vasco a través de un link on line (15\% de la muestra, 92\% respondió en castellano) y se hizo lo propio con trabajadores de México [6]. En este país la tarea de gestión y de campo estuvo a cargo de la coordinación de un programa universitario bajo la 
responsabilidad de una investigadora y su equipo (12\% de la muestra). Finalmente, se aplicó el F.I.N.O. en tres comunidades autónomas de España, en dos casos bajo la supervisión de una de las autoras de la escala en proyectos finales de maestría y en el tercer caso con supervisión de otra de las autoras del inventario en colaboración con investigadoras e investigadores del ámbito académico, empresarial y asociativo, 9\% de la muestra.

\section{Análisis de datos}

Se utilizó el programa SPSS versión 22 (Field, 2013; IBM, 2016) para tener una visión de la existencia de los factores de innovación en las organizaciones analizadas (descriptivos, medias y frecuencias de acuerdo $-5,6$ y 7 - con lo preguntado en el ítem). Para conocer la estructura interna de la escala se examinó la fiabilidad de cada dimensión y se realizaron análisis de componentes principales de máxima verosimilitud con rotación varimax sobre los ítems de la escala completa (70). Se seleccionaron aquellos ítems que presentaban un peso factorial igual o superior a 0.30 en un componente y cuando este incluyera 3 o más ítems - se explicita más adelante alguna excepción-y explicara, al menos el $2 \%$ de la varianza total (Carretero-Dios \& Pérez, 2005). Partiendo de los ítems seleccionados [7], se examinó la dimensionalidad del F.I.N.O llevando a cabo un análisis factorial confirmatorio (AFC) con MPLUS para calcular la fiabilidad mediante el coeficiente omega - siguiendo las recomendaciones de un revisor- (Revelle, 2016; Revelle \& Zimbarg, 2009) con el programa informático citado [8]. El AFC se hizo analizando los ítems que componían cada dimensión (por ejemplo, liderazgo positivo y de innovación) y postulando covarianza entre los factores de primer orden. Este procedimiento permitió calcular el coeficiente de fiabilidad omega en el caso de escalas con solo dos ítems - a excepción de auto-concepto creativo ya que no se podía suponer un factor de segundo orden $\mathrm{o}$ covarianza con inteligencia emocional y motivación- (ver también Wang \& Wang, 2012).
TABLA 1

Percepción de Innovación por características socio-demográficas

\begin{tabular}{|c|c|c|c|c|c|}
\hline & Total & Baja & Media & Alta & Valor-p \\
\hline \multicolumn{6}{|l|}{ Personas } \\
\hline Sexo, n (\%) & 889 & 313 & 310 & 266 & \\
\hline Hombres & $557(62.7)$ & $207(66.1)$ & $195(62.7)$ & $155(58.1)$ & $0.149^{\mathrm{c}}$ \\
\hline $\mathrm{n}$ & 829 & 274 & 302 & 253 & \\
\hline Edad (años), $\mathrm{M} \pm \mathrm{SD}^{\mathrm{b}}$ & $31.9 \pm 10.7$ & $32.1 \pm 11.4$ & $31.1 \pm 10.0$ & $32.6 \pm 10.6$ & $0.176^{\mathrm{d}}$ \\
\hline Escolaridad, n (\%) & 733 & 259 & 248 & 226 & \\
\hline Educación terciaria & $575(78.4)$ & $199(76.7)$ & $193(77.8)$ & $186(81.1)$ & $0.520^{c}$ \\
\hline Puesto gerencial, $\mathrm{n}(\%)^{\mathrm{a}}$ & 880 & 306 & 305 & 269 & \\
\hline si & $215(24.4)$ & $52(16.9)$ & $75(24.4)$ & $88(32.6)$ & $0.000^{\mathrm{c}}$ \\
\hline \multicolumn{6}{|l|}{ Financiación y apoyo } \\
\hline Sector, $\mathrm{n}(\%)^{\mathrm{a}}$ & 826 & 269 & 300 & 257 & \\
\hline Público & $451(54.6)$ & $163(60.4)$ & $164(54.8)$ & $124(48.2)$ & $0.060^{\circ}$ \\
\hline Privado & $353(42.7)$ & $98(36.5)$ & $128(42.6)$ & $127(49.5)$ & \\
\hline 3er. Sector (ongs, ...) & $22(02.7)$ & $8(03,0)$ & $8(02.7)$ & $6(02,2)$ & \\
\hline Empresa familiar, $\mathrm{n}(\%)^{\mathrm{a}}$ & 847 & 277 & 306 & 264 & \\
\hline si & $95(11.2)$ & $42(15.2)$ & $32(10.5)$ & $21(08.0)$ & $0.026^{\mathrm{c}}$ \\
\hline Tamaño, $\mathbf{n}(\%)^{\mathrm{a}}$ & 847 & 277 & 306 & 264 & \\
\hline Hasta 10 personas & $56(06.6)$ & $14(05.1)$ & $12(03.8)$ & $30(11.2)$ & $0.000^{c}$ \\
\hline De 11 a 50 personas & $122(14.4)$ & $50(18.0)$ & $42(13.8)$ & $30(11.2)$ & \\
\hline De 51 a 100 personas & $46(05.4)$ & $10(03.6)$ & $15(04.9)$ & $21(08.0)$ & \\
\hline De 101 a 500 & $123(14.5)$ & $29(10.3)$ & $43(14.1)$ & $51(19,2)$ & \\
\hline Más de 500 & $500(59.0)$ & $174(62.6)$ & $194(63.4)$ & $132(50.0)$ & \\
\hline Participación capital extranjero, $\mathrm{n}(\%)^{\mathrm{a}}$ & 847 & 277 & 306 & 264 & \\
\hline $\mathrm{si}$ & $159(18.8)$ & $46(16.5)$ & $69(22.4)$ & $44(16.6)$ & $0.106^{\mathrm{c}}$ \\
\hline
\end{tabular}

Fuente: elaboración propia

\section{Resultados}

\section{Percepción de innovación}

Se observa que en un alto grado hay acuerdo con el contenido de los ítems, de acuerdo con las medias, desviaciones típicas y porcentaje de varianza explicados de cada dimensión, que aparecen en la Tabla 2). En dicha tabla se presenta también la evaluación del grado en que los 17 jueces expertos evaluaron que el ítem reflejaba bien el constructo, en general, indicando acuerdo (primera columna) y los coeficientes de fiabilidad (el alfa que presupone unidimensionalidad $\mathrm{y}$ ausencia de covarianza entre errores y el omega que acepta multidimensionalidad). Finalmente, se muestra en la misma el peso del ítem en el factor en que se ubica y en el caso individual de cada subfactor.

Respecto a la totalidad de los ítems, se obtiene un valor de media muy superior a la teórica $(\mathrm{Mt}=$ 3.5). Las excepciones hacen referencia en el factor organizacional a se refuerza el intercambio con grupos de trabajo de otras organizaciones $(\mathrm{M}=$ 3.81 ítem de integración organizacional), los recursos tecnológicos y el conocimiento necesario para la innovación se ponen 
rápidamente a disposición de los trabajadores (M $=3.87$, ítem del factor recursos); en los factores individuales el salario que reciben $(\mathrm{M}=3.98$, motivación extrínseca). En menor medida se refuerza la comunicación con los usuarios y con otras organizaciones para hacer bien el trabajo, mejorarlo e innovar $(\mathrm{M}=4.00$ ítem de integración organizacional); la posibilidad de ganar poder e influencia a través de las tareas que realizan $(\mathrm{M}=$ 4.05 ítem de motivación extrínseca) y se aceptan las situaciones de incertidumbre y ambigüedad en relación con el desarrollo de nuevas ideas o su aplicación $(\mathrm{M}=4.07$ ítem de flexibilidad organizacional). El grado de acuerdo con el contenido en estos seis ítems es de valores entre $37 \%$ y $42 \%$. Si bien, el ítem dispongan de los recursos necesarios para efectuar el trabajo de modo adecuado (recursos organizacionales) tiene un índice de acuerdo solo del $21 \%$, su media es superior a la teórica y a la de estos otros ítems.

En cambio, es necesario destacar el alto acuerdo con que, en su propia organización, las personas perciben que: los responsables (jefes, superiores, otros) tienen con los trabajadores una relación de respeto mutuo $(\mathrm{M}=5.15$, ítem del factor liderazgo positivo, $68 \%$ de grado de acuerdo); las características de los puestos de trabajo de esta organización implica que las personas decidan cuando hacer un descanso $(\mathrm{M}=5.08,70 \%$ de grado de acuerdo) y tengan influencia sobre el orden en que se realizan las tareas ( $\mathrm{M}=5.03,68 \%$ de grado de acuerdo) ambos ítem de autonomía del factor características del rol laboral; las personas que trabajan en esta organización confían en su capacidad para ejecutar su trabajo de manera eficaz $(\mathrm{M}=5.01$, ítem del factor auto-eficacia laboral, $68 \%$ de grado de acuerdo); a las personas que trabajan en esta organización les motiva la seguridad de tener un trabajo $(\mathrm{M}=5$, ítem del factor motivación extrínseca, $67 \%$ de grado de acuerdo).

Otros ítems que presentan un grado de acuerdo superior al $60 \%$ son, los responsables (jefes, superiores, otros) de esta organización tienen con los trabajares una relación de contacto frecuente (64\% de acuerdo, $\mathrm{M}=4.88$, factor liderazgo positivo); los miembros de los grupos de trabajo de esta organización se preocupan de la calidad del trabajo realizado por su equipo, unidad de trabajo, otros $(65 \%$ de acuerdo, $\mathrm{M}=4.89$, factor orientación a la tarea y clima de excelencia); en los grupos de trabajo de esta organización existe una relación de confianza entre sus miembros (63\% de acuerdo, $M=4.81$, ítem de cohesión grupal), ...los objetivos están claramente definidos (63\% de grado de acuerdo, $M=4.88$ ); las personas que trabajan en esta organización confían en tener capacidad para desarrollar su trabajo empleando soluciones originales $(63 \%$ de grado de acuerdo, $\mathrm{M}=4.46$ ) y en los grupos de trabajo de esta organización predomina la colaboración entre las/os trabajadores $(62 \%$ de grado de acuerdo, $M=4.83$ ), ambos ítems de cohesión grupal.

En cuanto a la percepción global de innovación organizacional, las personas de esta encuesta ubican el nivel de innovación en su organización $(\mathrm{M}=4.14)$ por encima de la media teórica, al igual que la importancia atribuida a ella en su organización (4.38). Esta misma tendencia es percibida en otras organizaciones del sector (4.31 vs 4.54$)$. Por otro lado, la importancia otorgada a la innovación es percibida como superior en organizaciones similares a la suya $\mathrm{t}(940)=3.58, \mathrm{p}$ $<0.001$, al igual que el nivel de valoración t (947) $=3.13, \mathrm{p}<0.002$ de la innovación. Estos resultados estarían confirmando lo deseable y normativo del constructo y su práctica.

Estructura del inventario. Se contrastó la validez de contenido al inicio y al final de la denominada etapa 1 de validación, durante el año en curso. Se consultó a 20 expertos [9] de los países participantes (10 mujeres y 7 hombres devolvieron la encuesta). Se les solicitó que para cada ítem, afirmación o reactivo evaluaran de 0 a 5 si su contenido representaba el concepto en el que se enmarcaba. También se solicitó su colaboración para mejorar el ítem si la puntuación otorgada era inferior a 3 (ver Tabla 2). Para conocer el índice de acuerdo entre jueces se utilizó el coeficiente intraclase que es matemáticamente equivalente a los índices kappa y kappa ponderado (Prieto, Lamarca, \& Casado, 1998) de 9 jueces [10]. Se ha indicado que valores del CCI por encima de 0.75 representan una fiabilidad excelente, en este caso se obtuvo una medida única de $0.90 \mathrm{y}$ una promedio de 0.87 , IC $95 \%$ (0.72 - 0.96) con base en los 70 elementos. En general, se propusieron y aceptaron correcciones de forma y armonización del sujeto, verbo, etc., propuestas de división de 
ítems por considerarse algunos de ellos polisémicos, p.e., en el caso de la IE el ítem 17c, cambios de palabras a formas más coloquiales, especificación de alguna de las definiciones y evitación de redundancias (ver en la Tabla 2 ítems con asterisco $(*)$ con valoración menor a 3 ).

\section{TABLA 2}

Delimitación conceptual de la escala, acuerdo con el ítem (...en su organización...), fiabilidad y validez de contenido (jueces)

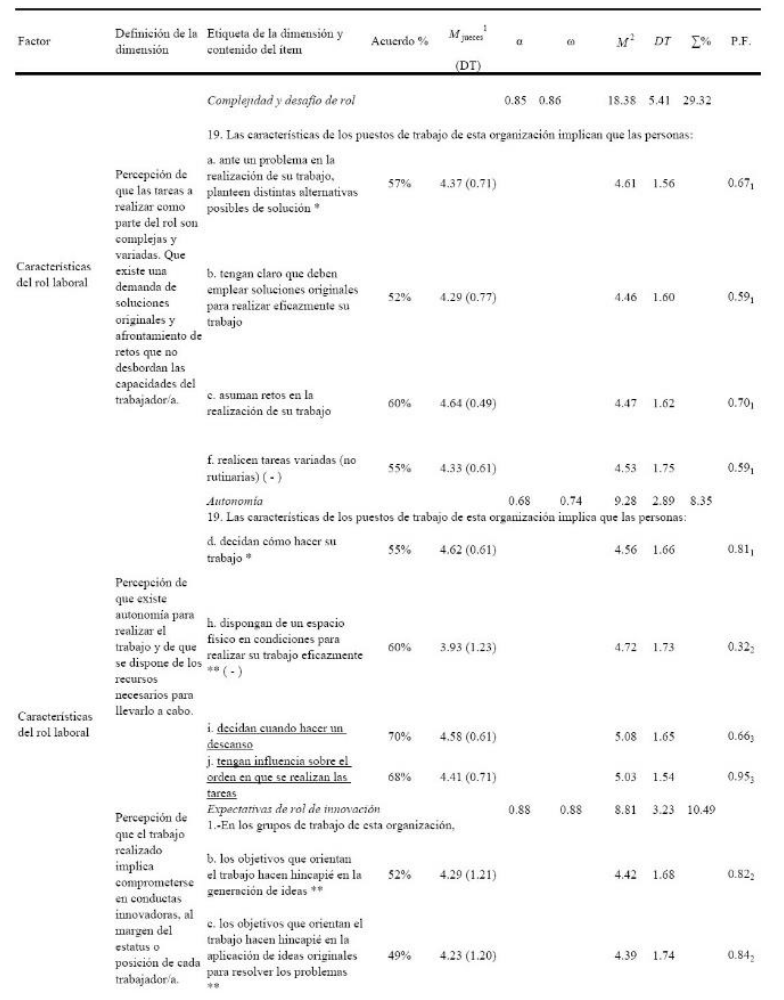

Fuente: elaboración propia.
TABLA 2 (cont.)

Delimitación conceptual de la escala, acuerdo con el ítem (...en su organización...), fiabilidad y validez de contenido (jueces)

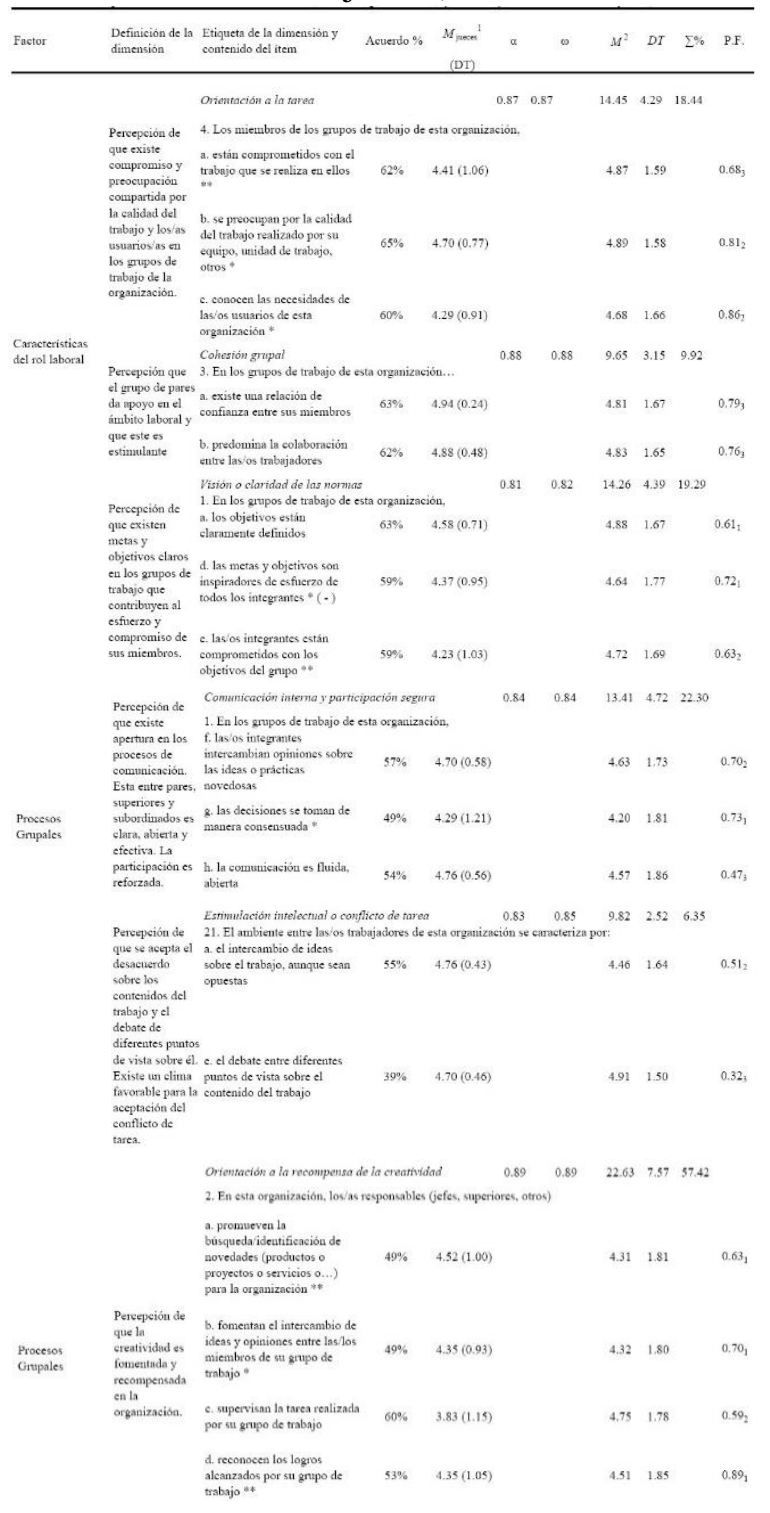


TABLA 2 (cont.)

Delimitación conceptual de la escala, acuerdo con el ítem (...en su organización...), fiabilidad y validez de contenido (jueces)

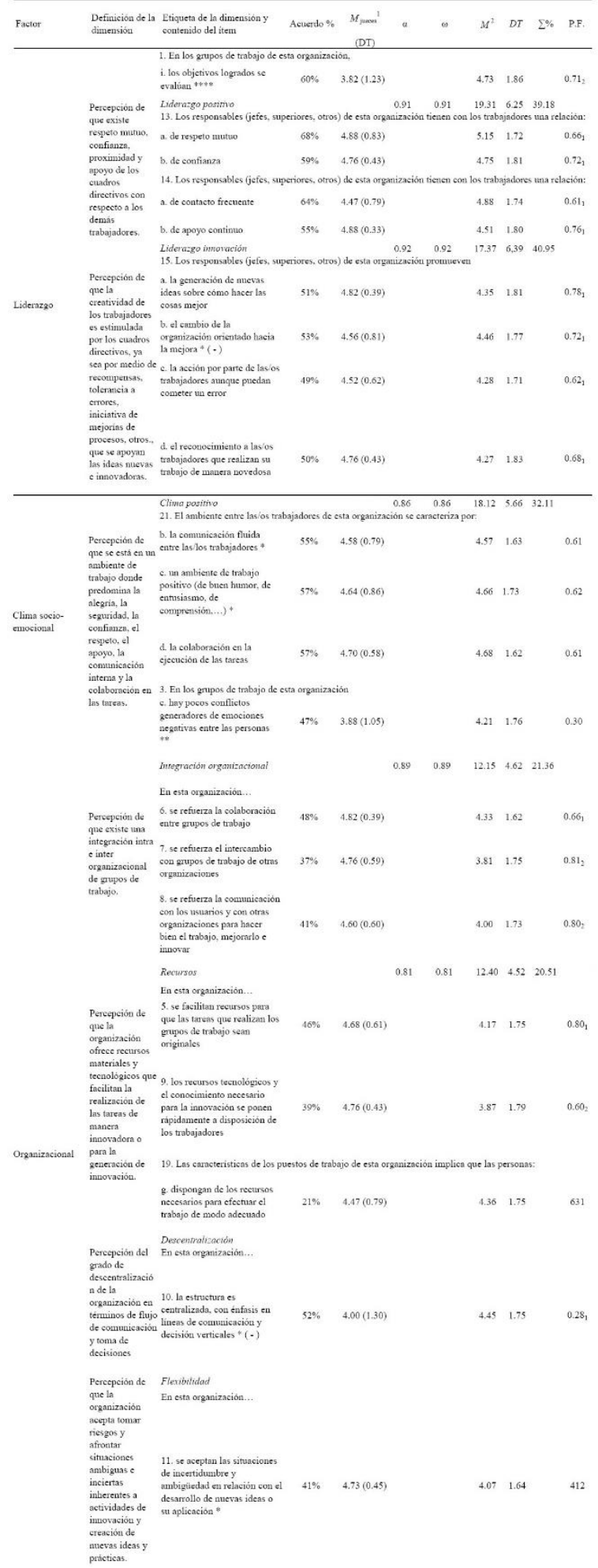

TABLA 2 (cont.)

Delimitación conceptual de la escala, acuerdo con el ítem (...en su organización...), fiabilidad y validez de contenido (jueces)

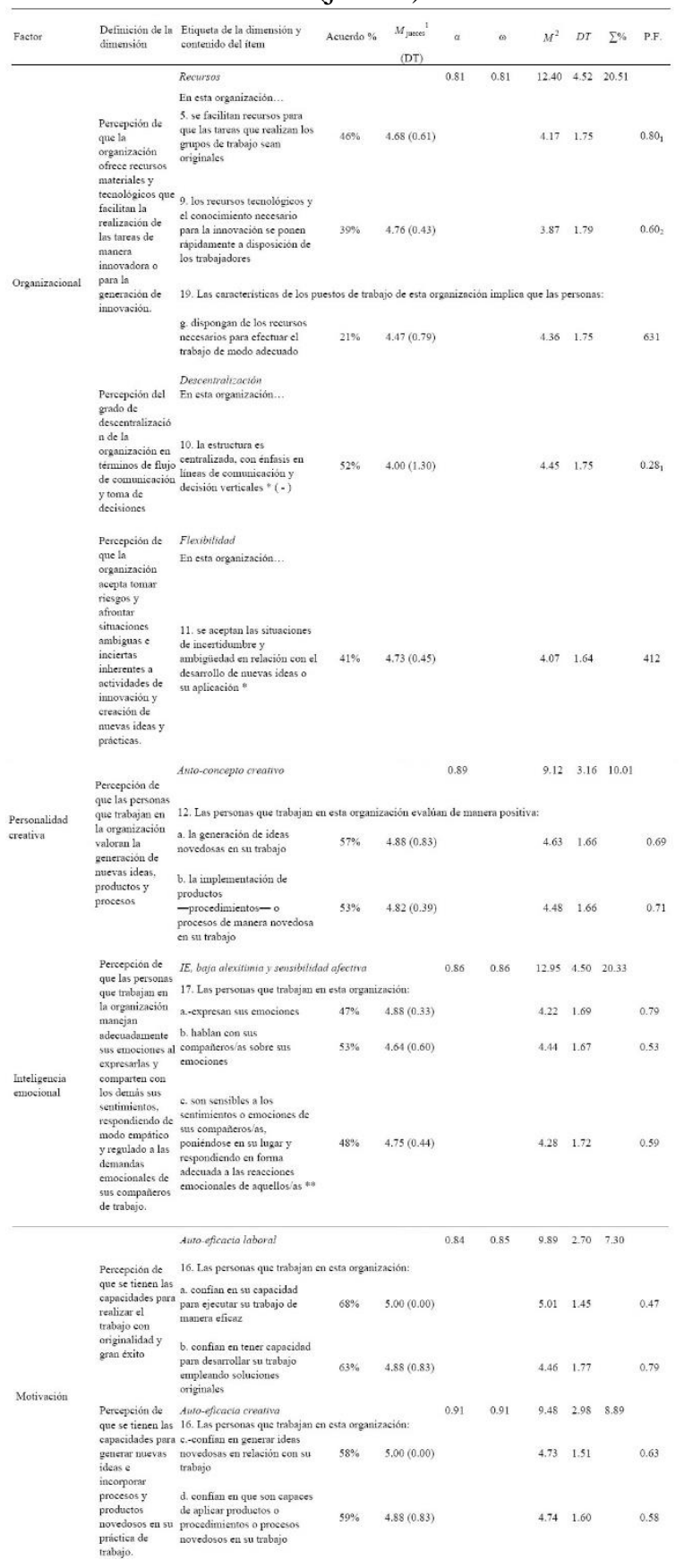


TABLA 2 (cont.)

Delimitación conceptual de la escala, acuerdo con el ítem (...en su organización...), fiabilidad y validez de contenido (jueces)

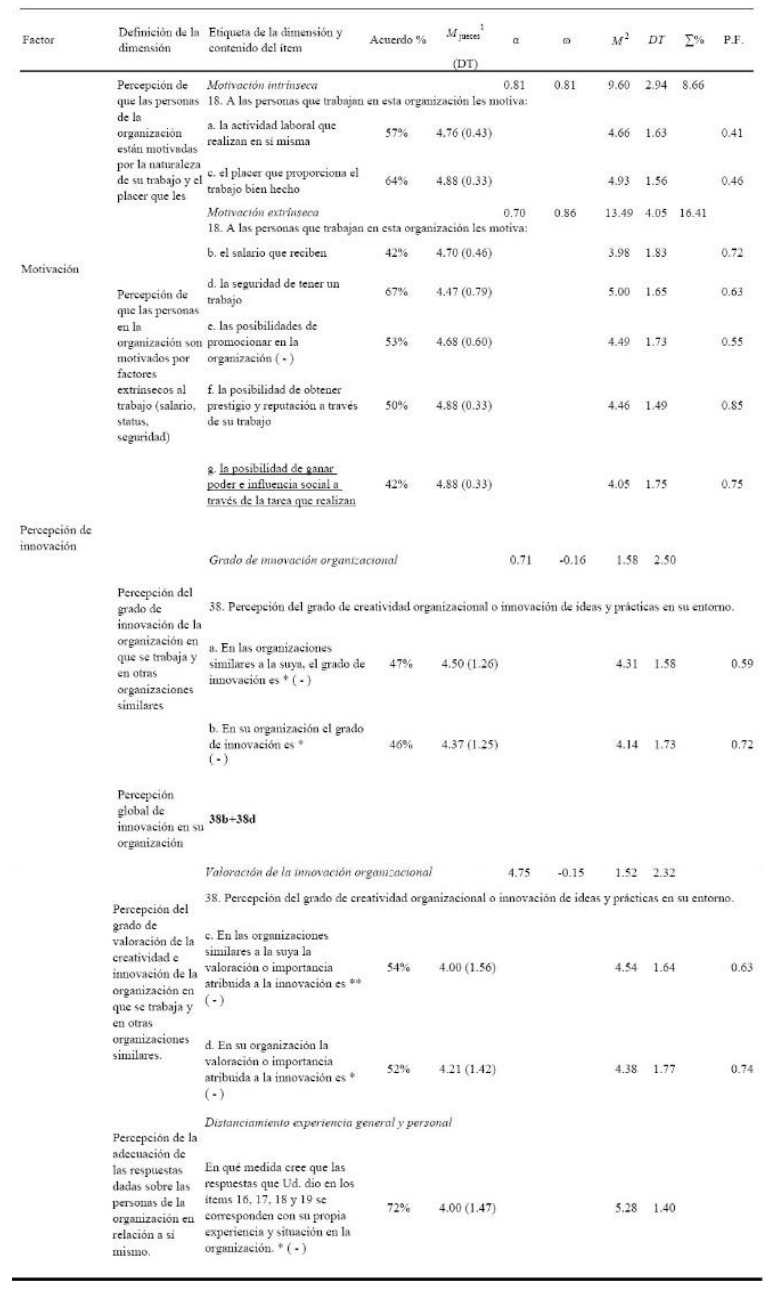

${ }^{1}$ Los jueces respondieron acuerdo con el ítem en una escala de respuesta $0=$ nada a $5=$ mucho.

${ }^{2}$ Las personas respondieron a la cuestión planteada en su organización con una escala de 1 $=$ nada aplicable a $7=$ muy aplicable. P.F.

$=$ Peso factorial. $*$ = cantidad de puntuaciones menores a 3 en el ítem. (-) al menos un juez no respondió a este ítem. $\sum \%=$ porcentaje de la varianza explicada. $\quad=$ ítem agregado en segunda recogida muestra. Fuente: elaboración propia

Se constata que al ampliar la muestra, las fiabilidades satisfactorias o muy satisfactorias $(\alpha>$ 0.70 ) se mantienen o aumentan, mientras que se ajustan los casos de fiabilidades como los de la dimensión motivación intrínseca (se había evidenciado que al eliminar el ítem de salario recibido la fiabilidad subía a 0.70 , sugiriendo que la remuneración era una variable per se y no vinculada a otras recompensas). Aquellas dimensiones que habían mostrado necesidad de agregar ítems en análisis anteriores mejoran su fiabilidad con los agregados (p.e., en autonomía [11] ítem i y j; ítem e en estimulación intelectual y conflicto de tarea y el e y g de motivación extrínseca) (ver Tabla

2). Los AFE permitieron contrastar en todos los factores, covarianza entre las dimensiones o un factor de segundo orden común, con la excepción del factor individual (que queda constituido por tres sub-factores: auto-concepto creativo, inteligencia emocional y motivación) y el de clima socioemocional. El AFC permitió constatar que las dimensiones reagrupaban adecuadamente los ítems y que los coeficientes de fiabilidad omega eran satisfactorios.

Para contrastar la validez predictiva o de criterio se utilizó el propio inventario de innovación (muestra total) y las escalas Setterlind de síntomas de estrés (muestra no militar ni México). Para la puntuación total se correlacionaron las puntuaciones de los factores psicosociales de la innovación con el indicador global de innovación contrastando la validez de criterio de cada dimensión (ver Tabla 3). Se constata en la muestra participante una asociación negativa y significativa con los síntomas de estrés de las escalas Setterlind (Istas, 2010), en forma significativa con conductuales $\mathrm{r}(425)=$ $0.17, \mathrm{p}<0.000$ y cognitivos $\mathrm{r}(425)=-0.12, \mathrm{p}<$ 0.005 , significando esto que a mayor percepción global de innovación, mayores síntomas de estrés [12]. Para contrastar la validez convergente se buscó conocer la asociación entre el factor de liderazgo del inventario F.I.N.O con la escala CELID que mide liderazgo transformacional percibido en relación al superior (Nader \& Castro Solano, 2009) en muestra militar. Los resultados indican una asociación positiva y significativa tanto con el liderazgo transformacional $\mathrm{r}(260)=0.21, \mathrm{p}<$ 0.001 como con el liderazgo transaccional $r(274)$ $=0.13, \mathrm{p}<0.01$. Cuando se analiza por dimensiones separadas (liderazgo positivo y liderazgo innovación) la asociación con el liderazgo transformacional, los resultados son muy similares mostrando algo más de fuerza $(0.20$ vs 0.21$)$ el liderazgo innovación. Comparando la percepción de 
innovación en relación con el puesto y la educación, se constata que quienes han ocupado algún cargo gerencial y mayor escolaridad son quienes perciben alta innovación mostrando la validez discriminante entre grupos - se puede pensar que las personas con mayor estatus e información tendrán una visión más positiva de la innovación-. Las empresas familiares, públicas y de menor tamaño (en comparación con la privada y las de mayor tamaño de personal) muestran menor percepción de innovación (ver Tabla 1). Finalmente, con la muestra total se calculó la puntuación de cada factor (rol laboral, procesos grupales, liderazgo, etc.) utilizándolas para predecir en una regresión múltiple la percepción de innovación.

Relación entre dimensiones y la percepción de innovación. Se realizaron correlaciones entre la percepción de los factores favorables a la innovación y la percepción del nivel de innovación de la organización por muestra participante (ver Tabla 3). Las correlaciones se promediaron mediante el procedimiento de Rosenthal y DiMatteo (2001).

\section{Figura 1}

Nivel percibido de innovación global ( $\mathrm{r}$ ponderada muestras de trabajadores, cadetes militares $y$ expertos). (Modelo 1).

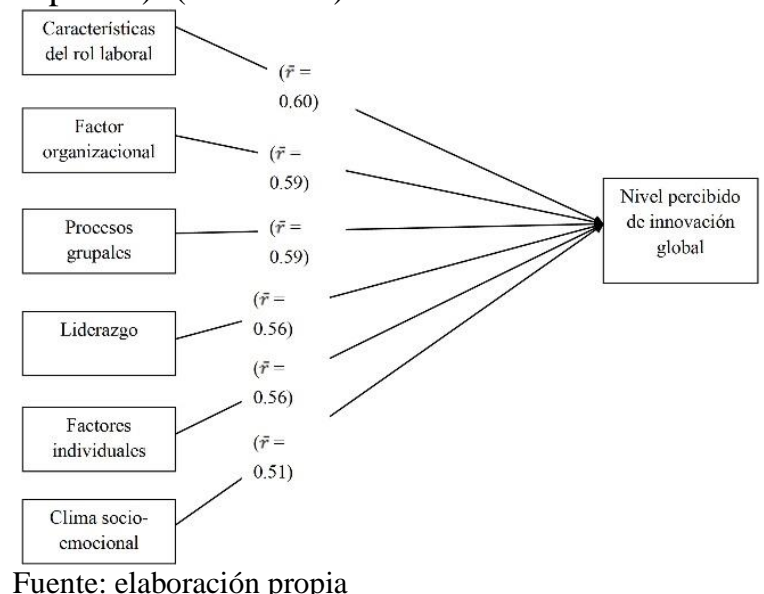

Fuente: elaboración propia

\section{TABLA 3}

Asociación entre los factores psicosociales de innovación incluyendo la variable criterio (muestra total) y entre factores y variable criterio (muestra participante)

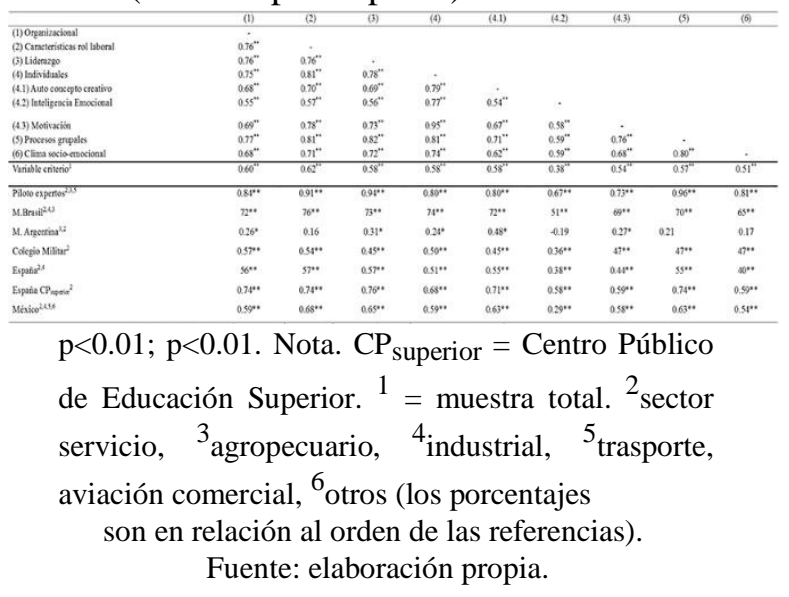

Las asociaciones entre factores psicosociales se dieron en el sentido esperado, aunque las de los expertos fueron más fuertes a pesar de los menores grados de libertad, sugiriendo su mayor validez (ver también resultados Brasil y España CPsuperior). Mientras la descentralización se asoció negativamente a la innovación en la muestra de trabajadores $(\mathrm{r}=-26)$ y cadetes $(\mathrm{r}=-0.24, \mathrm{p}<$ 0.000 ), lo hizo positivamente en la muestra piloto- expertos $(0.68, \mathrm{p}<0.004)$. Una revisión reciente mostró que la centralización no se asociaba negativamente a la innovación y que un cierto grado de jerarquía no inhibía la creatividad organizacional, por lo que este resultado no es incoherente con el estado actual de la evidencia (Damanpour \& Aaravind, 2012).

Según los meta-análisis, el factor organizacional y las características de rol laboral son los que predicen con más fuerza la innovación en las organizaciones coherentemente, en la muestra general de este estudio, el factor de rol laboral mostró una capacidad predictiva mayor. A través de tres regresiones múltiples se buscó contactar cuál era el peso de estas variables en el modelo analizado siendo la variable dependiente (VD) el grado y valoración de la innovación en la organización (ver Tabla 4). La regresión fue significativa en los tres casos explicando el 45, 49 y $50 \%$ de la varianza. Se confirmó la relación significativa de la innovación organizacional con los factores organizacionales y con los factores de rol laboral en las dos primeras regresiones, al igual que el puesto gerencial, el 
tamaño de la organización y la educación terciaria en la segunda regresión. La introducción del liderazgo en la tercera regresión quita fuerza al factor organizacional pasando a ser el tercero en importancia luego de este y ocupando el primer lugar las características del rol laboral. Los factores individuales (personalidad creativa, inteligencia emocional, motivación), los grupales y los de clima no mostraron influencia significativa, una vez controlados los factores citados. El puesto gerencial, el tamaño de la organización y en menor medida, la escolaridad terciaria muestran también su fuerza en la regresión, siendo las tres significativas.

\section{TABLA 4}

Regresión múltiple de la percepción global de innovación en las variables socio-demográficas y los factores de innovación

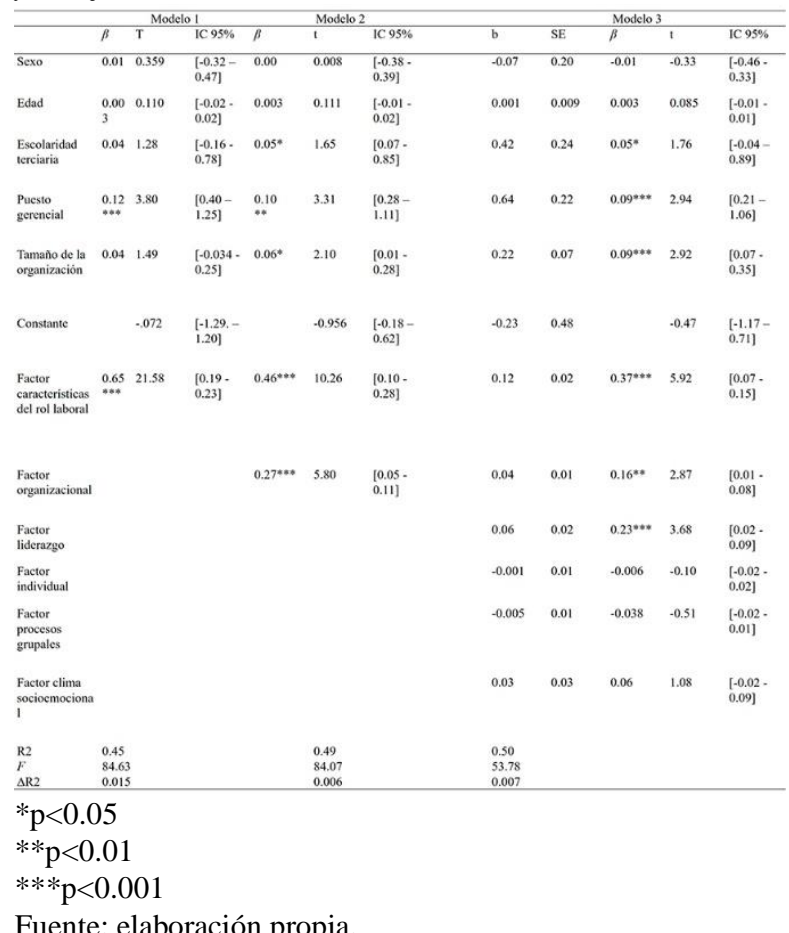

Fuente: elaboración propia.
Figura 2 Análisis mediacional de los predictores sobre el liderazgo con el factor global de innovación (efectos totales).

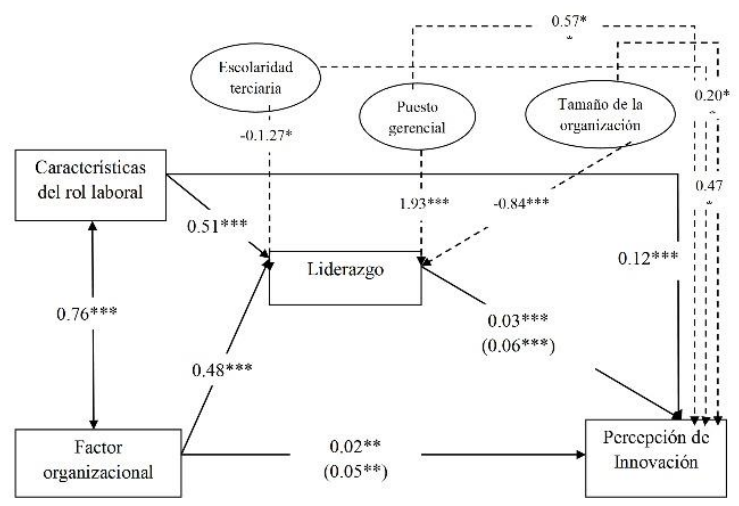

Fuente: elaboración propia

Se realizó un análisis mediacional a través del procedimiento de Preacher y Hayes (2008) para contrastar el modelo 2 postulado a partir del modelo 3 de regresión. Los factores de rol laboral $\mathrm{B}=0.031, \mathrm{DT}=0.008$, [IC bajo $=0.0147$, IC alto $=0.0494] \mathrm{y}$ organizacional $\mathrm{B}=0.029, \mathrm{DT}=0.008$, [IC bajo $=0.0136$, IC alto $=0.0465]$ muestran un coeficiente indirecto significativo a través del liderazgo que no incluía el cero en el intervalo de confianza. Los factores predictores ejercen influencia directa en la percepción global de innovación (como se había visto previamente en el modelo 1) e influencian al liderazgo que ejerce en este modelo como variable mediadora. El nivel educativo, puesto gerencial y tamaño de la organización —como covariables- ejercen influencia significativa en el liderazgo y en la percepción global de innovación. A menor educación y tamaño de la organización, mayor percepción de liderazgo positivo y de innovación. Ni el liderazgo ni el factor organizacional actúan como moderadores entre el rol laboral y la percepción de innovación organizacional al contrario de lo que se había postulado.

\section{Discusión y conclusiones}

La capacidad de innovación puede ser una estrategia de afrontamiento o un intento de mejorar el ambiente de trabajo (p.e., aumento del bienestar y posibles aumentos salariales, aprendizaje, crecimiento personal y 
reconocimiento, además de la satisfacción por la mejora), que aumenta la cohesión del grupo y puede invertirse en una mejor comunicación interpersonal (Ford \& Sullivan, 2004; West \& Farr, 2009). Los resultados de la muestra general indican que el nivel y la valoración de la innovación percibida en las organizaciones similares a la suya son altos, sugiriendo que la misma es una conducta deseable o norma social. También se informa que los factores psicosociales facilitadores de la innovación existen en un nivel medio-alto, percibiendo que la relación con los superiores es de respeto mutuo y contacto frecuente, aunque se da en menor medida que estos refuerzan la innovación.

En dicha muestra destaca la percepción positiva de la autonomía en el rol laboral, de autoeficacia individual y la consideración de que a las personas que trabajan en su organización les motiva la seguridad de tener un trabajo. Existe un alto grado de acuerdo en el factor procesos grupales, mayormente de orientación a la tarea y cohesión, mientras es ligeramente positiva en la búsqueda de novedades y el intercambio de ideas. La supervisión, el reconocimiento y la evaluación son percibidos más positivamente. Predomina una visión positiva del clima socio-emocional, mientras que hay una visión más crítica sobre la inteligencia emocional, concretamente se percibe que se habla con los compañeros sobre las emociones pero en menor medida se expresan, se es asertivo/a, empático/a en relación con los sentimientos o emociones de los compañeros/as y se regula adecuadamente las reacciones ante aquellos/as.

En relación con los superiores, los ítems de apoyo continuo y confianza reciben menos reconocimiento, y lo mismo sucede con la toma de decisiones consensuadas, el debate ante diferentes puntos de vista sobre el contenido del trabajo, el intercambio de ideas y opiniones entre los miembros de los grupos de trabajo. La integración organizacional como colaboración entre grupos de trabajo de la misma organización, el contacto con grupos de trabajo externos y con los usuarios se percibe minoritariamente. Hay un menor acuerdo con que se dispone de los recursos para realizar adecuadamente el trabajo y predomina una visión crítica tanto con la existencia de recursos para la innovación como con el salario.
En forma minoritaria se percibe que su organización acepta situaciones de incertidumbre y ambigüedad para innovar.

Las dimensiones de la escala F.I.N.O mostraron una consistencia interna adecuada y satisfactoria tanto en la muestra general como en las específicas. Las mismas se asocian a la innovación percibida en las organizaciones mostrando una adecuada validez. Sin embargo, tomando en cuenta las indicaciones de las y los expertos será necesario realizar algunos ajustes de forma, contenido y en algún caso analizar la propuesta de nuevos ítems. Se encuentran dimensiones del factor procesos grupales y del factor organizacional que aún pueden mejorar su consistencia interna, como por ejemplo, comunicación interna y participación segura, estimulación intelectual o conflicto de tarea y orientación a la recompensa de la creatividad.

Como se había hipotetizado, cuantos más factores psicosociales favorables a la innovación son percibidos en la organización, mayor es la percepción global de innovación organizacional, mostrándose una asociación positiva entre ellos. Además, confirmando la validez de criterio y discriminante entre grupos, las personas de puestos de alto estatus, de empresas de tamaño medio y grande y de empresas privadas informan de mayor percepción de innovación, coherentemente con estudios previos (Camisón \& Boronat, 2010). Las asociaciones también informan del coste de la innovación para el bienestar individual cuando muestran una relación significativa con los síntomas de estrés, específicamente los conductuales y cognitivos. Si bien los resultados sugieren que la innovación implica demandas que pueden ser agotadoras para las personas de esta muestra, se ha registrado anteriormente que también puede ser un recurso para el bienestar personal (Huhtala \& Parzefall, 2007).

Los resultados de las regresiones múltiples, en congruencia con la evidencia meta-analítica (da Costa, Páez, Sánchez et al., 2014), confirman que los aspectos de rol laboral y los organizacionales son más importantes para predecir la innovación que los individuales, de grupo y de clima socioemocional. Sin embargo, en esta muestra el liderazgo ejerce un peso superior al factor organizacional en la percepción de innovación global. Destacamos que este factor mostró 
validez convergente con otra escala en la muestra militar. Los resultados de mediaciones sugieren que la complejidad y desafío de rol, la autonomía y la expectativa de rol de innovación, así como la integración, los recursos y la flexibilidad organizacional refuerzan la percepción de factores de liderazgo, grupales e individuales y clima socioemocional positivo. Esto se puede interpretar en el sentido de que los roles laborales y organizaciones con estas características facilitan procesos de innovación.

El modelo 2 indica que el liderazgo puede ejercer el rol de mediador entre las características organizacionales, de rol y la percepción global de innovación. Ahora bien, no fue encontrado efecto de moderación sugiriendo que los factores psicosociales de la innovación actúan de igual forma en contextos organizacionales y de liderazgo de diferente calidad. También se contrastó la ausencia de relación positiva entre la innovación y la descentralización o negativa con la toma de decisión vertical por la dirección superior. Por otro lado, la revisión mas reciente muestra que la centralización se asocia negativa y significativamente en los estudios anteriores a 1990, pero no mostró asociaciones en los posteriores a 1990 (Damanpour \& Aaravind, 2012). Esto sugiere que la centralización, inevitable en cierto grado de complejidad y tamaño de empresas, no es un obstáculo para la innovación y el tamaño de la empresa, así como la existencia de reglas se asocia a ella (Camisón, \& Boronat, 2010; Damanpour \& Aaravind, 2012).

Que los factores organizacionales y de rol laboral sean los mejores predictores de la innovación así como el liderazgo cuando ejerce como tal, sugiere que para aumentar la creatividad serán más eficaces políticas de gestión organizacional como por ejemplo de cambios estructurales, de enriquecimiento del rol laboral, y de orientar las expectativas de rol hacia la innovación. La visión de recursos e integración organizacional en esta muestra era crítica, sugiriendo que son áreas en las que las mejoras son necesarias. En menor medida serían útiles cambios de estilo de liderazgo, aunque aumentar el apoyo continuo y la confianza facilitaría otros procesos innovadores. Un liderazgo transformacional, positivo y de innovación, enmarcado en una organización integrada y flexible que otorga recursos para la innovación, donde los roles laborales sean desafiantes y complejos, exista autonomía para realizar el trabajo en general y en particular implique comprometerse en conductas innovadoras facilitará la innovación mediando o creando procesos que permitan transformar las características estructurales organizacionales y de rol en creatividad aplicada - como indica su rol mediador-. En este sentido, los superiores deberían preocuparse por los riesgos y costos asociados con nuevas ideas y prácticas siendo responsables en última instancia del desempeño (Janssen, van De Vliert, \& West, 2004), mientras que la organización recuerda el costo de la innovación y apoya los errores y fracasos que la misma puede implicar para empleados y superiores (Cope, 2011).

Es de destacar que la selección de personal de alta auto-eficacia, motivación, personalidad creativa e inteligencia emocional y políticas orientadas a los procesos grupales y el clima serán menos influyentes. Las intervenciones deberían incluir por un lado, el apoyo organizacional a los errores y fracasos que la innovación puede implicar para empleados y superiores, y por otro, la mejora de las competencias emocionales (inteligencia y creatividad emocional, regulación adaptativa), creativas e innovadoras; manejo del estrés (sobrecarga de trabajo) y de la incertidumbre (Flintrop \& Vargas, 2014), que estas sean internalizadas en el auto-concepto, procesos grupales y la cultura organizacional. Se espera que a mayor competencia emocional, creativa e innovadora aumente la connotación positiva del cambio y la aceptación del riesgo.

Como limitaciones de este estudio se señalan que la muestra fue amplia, aunque de conveniencia, correlacionando auto-informes. Que las dimensiones o ítems vinculados directamente a la creatividad e innovación (p.e., expectativas de rol, orientación a la recompensa o liderazgo) se perciban con menor intensidad que los factores generales, podría sugerir que se tiene una visión más exigente e idealizada de la innovación que del funcionamiento cotidiano en su organización (Gondim et al., 2015). El que la percepción de determinados aspectos como p.e., motivación o auto-eficacia sean más favorables que la percepción de la descentralización o la flexibilidad organizacional podrían explicarse por ser los primeros más cercanos y concretos. Ahora bien, 
pese a estas limitaciones, la percepción de factores contextuales se asocia a la innovación más fuertemente que los factores individuales, en coherencia con estudios referenciados.

\section{Agradecimiento}

Este estudio se ha podido realizar gracias a la beca concedida a la primera autora, a la beca PSI201126315 y a la UFI 11/04

\section{Referencias}

Aboal, D., Angelelli, P., Crespi, G., López, A., Vairo, M., \& Pereschi, F. (2015). Innovación en Uruguay: diagnóstico y propuestas de políticas. Uruguay

+25, (documento de trabajo $\mathrm{N}^{\circ} 12$ ). Fundación Astur: Red Sur. Recuperado de http://www.cinve.org.uy/wp-content/up loads/2015/05/Documento-de-Trabajo-11. pdf

Adamsky, D. (2010). The culture of military innovation, the impact of cultural factors on the revolution in military affairs in Russia, the US, and Israel. Stanford: Stanford University Press.

Anderson, N., Poto\#nik, K., \& Zhou, J. (2014). Innovation and creativity in organizations: A state-of-the-science review, prospective commentary, and guiding framework. Journal of Management, 40, 1297-

1333. http://dx.doi.org/10.1177/01492063 14527128 .

Ashkanasy, N. M., \& Härtel, CH.E. (2014). Positive and negative affective climate and cultura: the good, the bad, and the ugly. En B. Schneider \& K. Barbera (Eds.), The Oxford handbook of organizational climate and culture (pp. 136-152). New York (USA): Oxford University Press. http://dx.doi.org/10.1093/oxfordhb/9780199 860715.013.0008.

Banco Interamericano de Desarrollo. BID

(2011). La necesidad de innovar, el camino hacia el progreso de América Latina y el Caribe. Recuperado de http:// craig.com.ar/biblioteca/La necesidad de innovar. Camino hacia el Progreso - BID.pdf
Barsade, M., Barsade, T., \& Licina, G. (2012). Organizational Development. En M. Mumford, Handbook of organizational creativity (pp. 667-703). London (UK): Elsevier.

Belbin, M. (2011). Equipos directivos, el por qué de su éxito o fracaso. Londres (UK): Elsevier.

Benito, S., Platero, M., \& Rodríguez, A. (2012). Factores determinantes de la innovación en las microempresas españolas: la importancia de los factores internos. Universia Business Review, 33, 104-121.

Binnewies, C., \& Gromer, M. (2012). Creativity and innovation at work: The role of work characteristics and personal initiative. Psicothema, 24, 100-105.

Bowen, F.E., Rostami, M., \& Steel, P. (2010). Timing is everything: a meta-analysis of the relationships between organizational performance and innovation. Journal of Business Research, 63, 1179-1185.

Bureau of European Policy Advises (BEPA) (2011). Empowering people, driving change: social innovation in the European Unión. Luxembourg: European Communities.

Buffet, M.A., Gervais, R.L., Liddle M., \& Eeckelaert, L. (2013). Well-being at work: creating a positive work environment. Literature Review. European Agency for Safety and Health at Work. Recuperado de https://osha.europa.eu/en/tools-and-pub lications/publications/literature_reviews/w ell-being-at-work-creating-a-positivework -environment

Cachia, R., Ferrari, A., Ala-Mutka, K., \& Punie, Y. (2010). Creative learning and innovative teaching: final report in the study on creativity and innovation in education in the EU member states. JRC scientific and technical reports: European commission. Recuperado de http://ftp.jrc.es/EURdoc/JR C62370.pdf

Camerer, C., \& Lovallo, D. (1999).

Overconfidence and excess entry: an experimental approach. The American Economic Review, 89 (1), 306-318. Cameron, K., \& Spreitzer, G. (Ed.) (2012). The 
Oxford handbook of positve organizational scholarship. New York (NY): Oxford University Press.

Camisón, C., \& Boronat, M. (2010). Estructuras organizativas, estrategias competitivas y ventajas estratégicas de las pyme. Mercados globalizados. Economía Industrial, 375, 89-100.

Cañibano, C. \& Castro, E. (2010). El Sistema español de innovación. En CSIC-UPV, Instituto de Gestión de la Innovación y del Conocimiento. Working paper 2010/09. Recuperado de http://www.ingenio.upv.es/sites/default/files /workingpaper/el_sistema_espa_ol_de_innovaci_n.pd $\mathrm{f}$

Caprile, M. (Coord.) (2012). Meta- analysis of gender and science research: synthesis report. Brussels: European Commision. Recuperado de http://ec.europa.eu/research/swafs/inde x.cfm?pg=library\&lib=gender_equality .

Carretero-Dios, H., \& Pérez, C. (2005). Normas para el desarrollo y revisión de estudios instrumentales. International Journal of Clinical and Health Psychology, 3, 521-551.

Confederación empresarial de Madrid- CEOE (CEIM) (2015). Memoria de la confederación. Recuperado de http://www.ceim.es/upload/file/publica ciones/memoria2015/ceim-2015-memoria. html

Cope, J. (2011). Entrepreneurial learning from failure: an interpretative phenomenological analysis. Journal of Business Venturing, 26, 604-623. http://dx.doi.org/10.1016/j.jbus vent.2010.06.002 .

Cooper, A., Dunkelberg, W., \& Woo, C. (1988). Entrepreneurs'perceived chances for success. Journal of Business Venturing, 3 (2), 97-108. http://dx.doi.org/10.1016/08 83-9026(88)90020-1

Cotec (2016). Informe Cotec 2016: Innovación en España. Recuperado de http://cotec.es/ informe-cotec/

Crespi, G., Navarro, J.C., \& Zúñiga, P. (2010). Ciencia, tecnología e innovación en América Latina y el Caribe: un compendio estadístico de indicadores. Banco Interamericano de Desarrollo (BID). Recuperado de http://www.iadb.org/es/temas/competiti vidad-tecnologia-e-innovacion/publicacio nes-e-investigacion, 7758.html

Crespi, G., \& Zúñiga, P. (2010). Innovation and productivity: evidence from six Latin American countries. IBD working paper. Recuperado de http://www.iadb.org/res/pu blications/pubfiles/pubIDB-WP-218.pdf

Csikszentmihalyi, M. (2003). Fluir en los negocios: liderazgo y creación en el mundo de la empresa. Barcelona (BC): Kairós.

Csikszentmihalyi, M. (2011). Creatividad, el fluir y la psicología del descubrimiento y la invención. Barcelona (BC): Paidós.

da Costa, S., Páez, D., Oriol, X., \& Unzueta, C. (2014). Regulación de la afectividad en el ámbito laboral: validez de las escalas de heteroregulación EROS y EIM. Journal of Work and Organizational Psychology, 30 , 13-22. http://dx.doi.org/http://dx.doi.org/ $10.5093 /$ tr2014a2

da Costa, S., Páez, D., Sánchez, F., Garaigordobil, M., \& Gondim, S. (2015). Personal factors of creativity: a second order meta-analysis. Journal of work and organizational Psychology, 31, 165-173.: http://dx.doi.org/10.1016/j.rpto. 2015.06.002

da Costa, S., Páez, D., Sánchez, F., Gondim, S., \& Rodríguez, M. (2014). Factores favorables a la innovación en las organizaciones: una integración de meta-análisis. Journal of Work and Organizational Psychology, 30, 67-74. http://dx.doi.org/10.1016/j.rpto.2014.06.006 da Costa, S., Páez, D., Hermosilla, D., Gondim, S., Oriol, X., Rodríguez, M., Torres, A. (junio de 2016). Percepción de liderazgo e innovación en las organizaciones: dosestudios. Trabajo presentado en el Congreso Internacional de Psicología del Trabajo y Recursos Humanos. Madrid, España.

da Costa, S., Páez, D., \& Sánchez, F. (julio de 2016). Creatividad y bienestar en contextos de trabajo. En S. Gondim, X. Oriol, \& S. da Costa. Simposio: regulaçao, criatividade e 
bem-estar: explorando perspectivas de abordagem em contextos de trabalho $y$ deportivos. Simposio llevado a cabo en el Congreso VII CBPOT, Brasilia.

da Costa, S., Sánchez, F., \& Páez, D. (2016). Confianza en las organizaciones y su relación con la creatividad y la innovación percibida. En M. Bargsted., H. Acosta., C.M. Alcover \& S. da Costa. Simposio: Confianza en las organizaciones: antecedentes de su impacto en el comportamiento organizacional a nivel de equipos y personas y su relación con la cultura organizacional y la innovación. Congreso Internacional de Psicología del Trabajo y Recursos humanos. Celebrado en Madrid, España los días 2 y 3 de junio de 2016. Presentación no publicada.

Damanpour, F., \& Aaravind, D. (2012), Organizational structure and innovation revisited from organic to ambidextrous structure. In M. Mumford (Ed.), Handbook of Organizational Creativity (pp. 483-513). London: Academic Press.

Davis-Blake, A., \& Broschak, J. (2009). Outsourcing and the changing nature of work. Annual Review of Sociology, 35, 321-340.

De Neys, W. (2010). Heuristic bias, conflict, and rationality in decision-making. En B.M. Glatzeder, V. Goel, \& A. von Müller (Eds.), Towards a theory of thinking (pp. 22-33). Berlin, Germany: Springer-Verlag.

Departamento de Educación, Política Linguiística y Cultura del Gobierno Vasco (Hezkuntza). Formación e innovación (2012). ¿Hacia dónde va la innovación? Programa de Gobierno 2012-2016, Plan Heziberri, 2020 y otros. Recuperado de http://www.hezkun tza.ejgv.euskadi.eus/r43-2459/es/contenid os/informacion/dig/es_presenta/presentaci on.html

Dutta, S., Lanvin, B., \& Wunsch- Vincent, S. (2015). The global innovation index: effective innovation policies for development, Fontainebleau, Ithaca, and Geneva. Recuperado de https://www.globalinnovationindex.org/user files/file/reportpdf/GII-2015-v5.pdf

European Union (EU), política regional (2009). Creatividad e innovación, motor de competitividad en las regiones. Panorama inforegio, 29, 3-29.

European commission (2015). En Innovation Union a Europe 2020 iniciative. Recuperado de http://ec.europa.eu/research/innovationunion/index_en.cfm?pg=keyd ocs

European Commission (EC) (2016). European innovation scoreboard. Recuperado de http://ec.europa.eu/growth/industry/innovati on/facts-figures/scoreboards_en

European Union (EU) (2016). Europe 2020 indicators, research and development. Eurostat Statistics Explained. Recuperado de http://ec.europa.eu/eurostat/statisticsexplained/index.php/Europe_2020_indicator s_-_research_and_development

European Commission (EC) (2017). Entrepreneurship in Education. Recuperado de http://ec.europa.eu/educati on/policy/strategic-framework/entreprene urship_en

Fagerberg, J., Mowery, D., \& Richard, N. (Eds.). (2009). The Oxford handbook of innovation. New York (NY): Oxford University Press.

Faleg, G. (2016). The implications of Brexit for the EU's common security and defense policity. Recuperado de https://www.ceps.eu/publications

Fiedler, K., \& von Sydow, M. (2015). Heuristics and biases: beyond tversky and Kahneman's (1974) judgment under uncertainty. In M.W. Eysenck \& D. Groome, Cognitive psychology: revisiting the classical studies (pp. 146-161). Los Angeles (UK): Sage.

Field, A. (2013). Discovering statistics using IBM SPSS statistics. London: Sage Publications.

Flintrop, J., \& Vargas, O. (2014). Psychosocial risk in Europe: prevalence and strategies for prevention. Luxembourg: EU-OSHA. Recuperado de https://osha.europa.eu/en/tools-and-pub lications/publications/reports/psychosocialrisks-eu-prevalence-strategiesprevention/view

Foley, R. (2012). A case study in horizontal military innovation: the german army, 19161918. The Journal of Strategic Studies, 35 (6), 799-827.

Ford, C.M., \& Sullivan, D.M. (2004). A time for everything: how the timing of novel 
contributions influences proyect team outcomes. Journal of Organizational Behavior, 25 (2), 279-292.

Fundación Botín. (2012). Good morning creativity! Awakening human potential through education. (Botín Foundation report). Recuperado de http://www.centrob otin.org/oedihg287ddy278_uploads/web_1/p ersonales/Creatividad/buenosdiascreati vidad.pdf Fun

Fundación Botín. (2014). Artes y emociones que potencian la creatividad. (Informe Fundación Botín). Recuperado de http:// www.centrobotin.org/oedihg287ddy278_upl oads/web_1/personales/Creatividad/2014Inf orme Creatividad ES.pdf

Garaigordobil, M. (2003). Intervención psicológica para desarrollar la personalidad infantil: juego, conducta prosocial y creatividad. Madrid: Pirámide.

Gardner, H., \& Csikszentmihalyi, M. (2002). Buen trabajo: cuando ética y excelencia convergen. Barcelona: Paidós.

Generalitat de Catalunya (Gencat) (2012). La innovació a lempresa. Recuperado dehttp://inicia.gencat.cat/inicia/es/innovacio. do

Gigerenzer, G., \& Gaissmaier, W. (2011). Heuristic decisión making. Annual Review of Psychology, 62, 451-482. http://dx.doi.org/10.1146/annurev-psych120709-145346.

Gondim, S., Loiola, E., Andrade de Morais, F., da Costa, S., Páez, D., Rodriguez, M.,... Mourao, L. (2015). Creativity and innovation as defined by workers. REAd, 82 (3), 549575. http://dx.doi.org/10.1590/

1413-2311.0162015.55629

González, J.L., Navarro, M., \& Peña, I. (2010). Internacionalización de empresas innovadoras jóvenes en España. Revista Europea de Dirección y Economía de la Empresa, 19 (2), 61-82.

González, J.L., \& Peña. I. (2007). Determinantes de la capacidad de innovación de los negocios emprendedores en España. Economía Industrial, 363, 129-147.

Hammond, M., Neff, N., Farr, J., Schwall, A., \& Zhao, X. (2011). Predictors of IndividualLevel Innovation at Work: A Meta-Analysis.
Psychology of Aesthetics, Creativity, and the Arts, 5, 90-105, http:// dx.doi.org/10.103720018556.

Hennesey, B., \& Amabile, T. (2010). Creativity.

Annual Review of Psychology, 61,

569-598. http://dx.doi.org/0.1146/annurev. psych.093008.100416

Hervás, G., \& Vázquez, C. (2013). Construction and validation of a measure of integrative well-being in seven languages: The Pemberton Happiness Index. Health Qual Life Outcomes , 22 , 11-66. http://dx.doi.or $\mathrm{g} / 10.1186 / 1477-7525-11-66$

Hermosilla, D., Amutio, A., da Costa, S., \& Páez, D. (2016). El liderazgo transformacional en las organizaciones: variables mediadoras y consecuencias a largo plazo. Journal of Work and Organizational Psychology, 32 (3), 135143. http://dx.doi.org/10.1016/j.rp to.2016.06.003

Horowitz, M. (2010). Nostate actors and the diffusion of innovations: the case of suicide terrorism. International Organization, 64 (1), 33-64. http://dx.doi.org/10.1017/S002 0818309990233

Hubert, A. (2010). Empowering people, driving change: social innovation in the European Union.

Recuperadode

https://ec.europa.eu/migrant-

integration/librarydoc/empowering-peopledriving-c hange-social-innovation-in-theeuropean- union

Huhtala, H., \& Parzefall, M. (2007). A review of employee well-being and innovativeness: an opportunity for a mutual benefit. Creativity and Innovation Management, 16 (3), 299$306 . \quad$ http://dx.doi.org/10.1111/j.14678691.2007.00442.x

Huhtala, M., Kangas, M., Lämsä, A.M., \& Feldt, T. (2013). Ethical managers in ethical organizations? The leadership-culture connection among Finnish managers. Leadership \& Organization Development Journal, $34 \quad$ (3), 250-270. http://dx.doi.org/10.1108/014377313113266 84

Hülsheger, U., Anderson, N., \& Salgado, J. (2009). Team-Level Predictors of Innovation at Work: A Comprehensive Meta-Analysis Spanning Three Decades of Research. 
Journal of Applied Psychology, 94, 11281145. http://dx.doi.org/103720015978.

Hunter, S., Bedell, K., \& Mumford, M.D. (2007). Climate for creativity: a quantitative review. Creativity Research Journal, 19, 69-90. http://dx.doi.org/10.1080/104004107093368 83.

IBM (2016). Guía breve de IBM SPSS statistics 22 Recuperado de ftp://public.dhe.ibm.com/software/analytics/ spss/documentation/statistics/22.0/es/client/ Manuals/IBM_SPSS_Statistics_Brief_Guide .pdf

Instituto Nacional de la juventud (Injuve) (2015). Education a policy Outlook 2015. Recuperado de http://www.injuve.es/observatorio/noticia/ed ucation-policy-outlook-

2015\#sthash.n2BJg4us.dpuf

Innobasque (Berrikuntzaren euskal agenzia / agencia vasca de la innovación) (2016). Plan de Ciencia, tecnología e innovación 2020. Recuperado de https://www.irekia.euskadi.eus/uploads/attac hments/5585/PCTI_Euskadi_2020.p df

Janssen, O., van de Vliert, E., \& West, M. (2004).

The bright and dark sides of individual and group innovation: a Special Issue introduction. Journal of Organizational Behavior, 25, 129-145. http://dx.doi.org/10.1002/job.242.

Jiménez, S., \& Bruzzo, S. (2016). Ciencia, tecnología e innovación para el desarrollo. Serie informe económico, 256, 1-29. Recuperado de http://lyd.org/wp-content/u ploads/2016/05/SIE-256-Ciencia-Tecnolo gia-e-Innovacion-para-el-desarrolloAbril2016.pdf

Jordan, J. (2015). Cultura organizativa e innovación militar: el caso de las fuerzas de defensa de Israel. Estudios de seguridad $\begin{array}{llll}\text { nacional, } & 1 & \text { (1), } & \text { 17-40. }\end{array}$ http://dx.doi.org/10.18847/1.1.2

Kahneman, D. (2012). Pensar rápido, pensar despacio. Barcelona (ES): Debate.

Landström, H., \& Lohke, F. (Eds.) (2010). Historical foundations of entrepreneurial research. Cheltenham (UK): EE.

Larracoechea, I. (27 de octubre de 2013). La innovación como motor de la recuperación. Diario El País. Recuperado el 27 de octubre de 2013 de http://economia.elpais.com/economia/2013/ 10/25/actualidad/1382698176_594293.html

Ma, H. (2009). The Effect Size of Variables Associated With Creativity: A MetaAnalysis. Creativity Research Journal, 21, 30-42.

http://dx.doi.org/10.1080/104004108026334 00

Noe, R., Clarke, A., \& Klein, H. (2014). Learning in the twenty-first-century workplace. Annual Review of Organizational Psycholy and Organizational Behavior, 1, 245-275. http://dx.doi.org/10.1146/annure vorgpsych-031413-091321

Nonaka, I., \& Toyama, R. (2005). The theory of the knowledge-creating firm: subjectivity, objectivity and synthesis. Industrial and corporate change, 14 (3), 419-436. http://dx.doi.org/10.1093/icc/dth058

Miller, R., Mark, N., \& Michaelson, J. (2008). Innovation and well-being. (InnovationIndex working paper) NESTA. Recuperado de https://www.nesta.org.uk/sites/default/f iles/kcfinder/files/6.2.InnovationandWellb eingMilleretal.pdf

Ministerio da Ciência, Tecnologia, Inovações e Comunicações (Mincyt). (2013). Estratègia Nacional de Ciencia, Tecnología e Inovaçao 2016-2019. Recuperado de http://www.mcti.gov.br/publicacoes

Ministerio de Ciencia, tecnología e innovación productiva (Mincyt). Presidencia de la nación (2015). Plan en acción "Argentina innovadora 2020". Recuperado de http://www.mincyt.gob.ar/informes/pla nen-accion-argentina-innovadora-202011634

Ministerio de Economía y Competitividad (Mineco). (2013) Estrategia española de ciencia y tecnología y de innovación 20132020. Recuperado de http://www.idi. mineco.gob.es/stfls/MICINN/Investigacio n/FICHEROS/Estrategia_espanola_cienci a_tecnologia_Innovacion.pdf

Moscovici, S. (1976). Social influence and social 
change. London: Academic Press.

Mumford, M., Hester, K.S., \& Robledo, I.C. (2012). Creativity in organizations: importance and approaches. En M. Mumford (Ed.), Handbook of organizational creativity (pp. 3-16). London: Elsevier.

Naciones Unidas/UNDP/Unesco (2014). Creative economy report 2013 special edition, widening local development pathways. Recuperado de http://www.unesco.org/culture/pdf/crea tiveeconomy-report-2013.pdf .

Navarro, J.C., \& Olivares, J. (2016). La políticade innovación en América Latina y el Caribe, nuevos caminos. Washington, DC: BID. Recuperado de http://repositorio.minedu.gob.pe/bitstre am/handle/123456789/4604/Lapolíticadei nnovaciónenAméricaLatinayelCaribenuev oscaminos.pdf?sequence $=1$

O`Boile, E.H., Humphrey, R. H., Pollack, J.M., Hawver, T.H., \& Story, P.A. (2011). The relation between emotional intelligence and job performance: a meta-analysis. Journal of Organizational Behavior. 32, 788-818. http://dx.doi.org/10.1002/job.714.

Oriol, X., Amutio, A., Mendoza, M., da Costa, S., \& Miranda, R. (2016). Emotional creativity as predictor of intrinsic motivation and academic engagement in university students: the mediating role of positive emotions. Frontiers in Psychology. http://dx.doi.org/10.3389/fpsyg.2016.01243

Ortiz, S., Navarro, C., García, E., Ramis, C., \& Manassero, A. (2012). Validación de la versión española de la escala de trabajo emocional de Frankfurt. Psicothema, 24 (2), 337-342.

Organisation for Economic Co-operation and Development (OECD) (2005). Oslo Manual: Guideliness for collecting and interpreting innovation data. OECD/EC.

Patterson, F., Kerrin, M., \& Gatto-Roissard, G. (2009). Everyday innovation, how the enhance innovative working employees and organizations. (Research report). Nesta. Recuperado de https://www.nesta.org.uk/s ites/default/files/everyday_innovation.pdf

Pelkmans, J., \& Renda, A. (2014). Does EU regulation hinder or stimulate innovation? Centre for European Policity Studies. Brussels, Belgium. Recuperado de https://www.ceps.eu/publications/does-euregulation-hinder-or-stimulate-innovati on

Peña-Sarrionandia, A., Mikolajczak, M., \& Gross, J.J. (2015). Integrating emotion regulation and emotional intelligence traditions: a metaanalysis. Frontiers in Psychology, 6, 160, 127.

http://dx.doi.org/10.3389/fpsyg.2015.00160

Pisano, U., Lange, L., \& Berger, G. (2015). Social innovation in Europe: an overview of the concept of social innovation in the context of European initiatives and practices. ESDN Quarterly Report, 36. Recuperado de http://www.sdnetwork.eu/quarterly\%20repo rts/report\%20files/pdf/2015-AprilSocial_Innovation_in_Europe.pdf

Preacher, K. J., \& Hayes, A. F. (2008). Asymptotic and resampling strategies for assessing and comparing indirect effects in multiple mediator models. Behavior Research Methods, 40, 879-891.

Plan Uruguay 2015-2020 (2015). Vamos Uruguay. Recuperado de http:// plan.vamosuruguay.com/

Revelle, W. (2016). psych: Procedures for Personality and Psychological Research. (R package version 1.6.4.) [Computer Software]. North Western University, Evanston. Recuperado de http://cran.r-proj ect.org/web/packages/psych/ .

Revelle, W., \& Zinbarg, R. (2009). Coefficients alpha, beta, omega, and the glb: coments on sijtsma. Psychometrika, 74, 145-154. http: //dx.doi.org/0.1007/S11336-008-9102-Z

Prieto, L., Lamarca, R., \& Casado. A. (1998). La evaluación de la fiabilidad en las observaciones clínicas: el coeficiente de correlación intraclase. Medicina clínica, 110 (4), 142-145.

Rauch, A., \& Frese, M. (2007). Let's put the person back into entrepreneurship research: a meta-analysis on the relationship between business owners' personality traits, business creation, and success. European Journal of Work and Organizational psychology, 16 (4), 353-385. http://dx.doi. org/10.1080/13594320701595438. 
Riivari, E., Lämsä, A., Kujala, J., \& Heiskanen, E. (2012). The ethical cultura of organisations and organisational innovativeness. European Journal of Innovation Management, 5 (3), 310-331. http://dx.doi.org/10.1108/146010 61211243657.

Robbins, S, \& Judge, T. (2016). Essentials of organizational behavior. England (UK): Pearson Education.

Robbins, S. (2008). Fundamentos do comportamento organizacional. Sao Paulo (SP): Pearson.

Rosenbusch, N. Brinckmann, J., \& Bausch, A. (2011). Is innovation always beneficial? A meta-analysis of the relationship between innovation and performance in SMEs. Journal of Business Venturing, 26 (4), 441457. http://dx.doi.org/10.1016/j.jbusv ent.2009.12.002

Romero, M.J. (2014). El impacto económico de la innovación: 10 razones por las que innovar. Madrid: Clarke, Modet \& Co.

Rosenthal, R., \& DiMatteo, R. (2001). Meta-analysis: Recent Developments in Quantitative Methods for Literature Reviews. Annual Review of Psychology, 52

, 59-82. http://dx.doi.org/10.1146/annurev. psych.52.1.59

Sánchez Bueno, M.J. (2008). El proceso innovador y tecnológico: estrategias y apoyo público. La Coruña: Netbiblo.

Sarooghi, H., Libaers, D., \& Burkemper, A. (2015). Examining the relationship between creativity and innovation: a meta- analysis of organizational, cultural and environmental factors. Journal of Business Venturing, 30 , 714-731. http://dx.doi.org/ 10.1016/j.jbusvent.2014.12.003

Scarpinelli, L. (2013, septiembre 15). Innovación y desarrollo: contradicciones en la política oficial. Diario La Nación. Recuperado de http://www.lanacion.com.ar/1619890-c ontradicciones-en-la-politica-oficial

Scott, R. (2008). Institutions and organizations: ideas and interests. Los Angeles (CA): Sage Publications.

Schumacher, E.G., \& Wasieleski, D. M. (2013). Institutionalizing ethical innovation in

| Universitas Psychologica | V. 15 | No. 4 | Octubre-Diciembre | 2016 | organizations: an integrated causal model of moral innovation decision processes. Journal Buiness Ethics, 113 (1), 15-37. http://dx.doi.org/10.1007/s10551-012-12777.

Sistema integrado de información sobre investigación científica, desarrollo tecnológico e innovación (SIICyT) (2013). Plan Nacional de Desarrollo 2013-2018. Recuperado de http://pnd.gob.mx/

Song, M., Podoynitsyna, K., Van Der Bij, H., \& Halman, J. (2008), Success Factors in New Ventures: A Meta-analysis. Journal of Product Innovation Management, 25, 7-27. http://dx.doi.org/10.1111/j.15405885.2007.00280.x

Unesco (2016). Revisión comparativa de iniciativas nacionales de aprendizaje móvil en América Latina. Los casos de Colombia, Costa Rica, Perú y Uruguay. Paris (FR): ONU.

Vilagut, G., Ferrer, M., Rajmil, L., Rebollo, P., Permanyer-Miralda. G., Quintana, J., Santed, R., Valderas, J., Rivera, A., DomingoSalvany, A. \& Alonso, J. (2005). El Cuestionario de Salud SF-36 español: una década de experiencia y nuevos desarrollos. The Spanish version of the Short Form 36 Health Survey: a decade of experience and new developments. Gac Sanit, 19 (2), 13550 .

Villamizar, G. (2012). La creatividad desde la perspectiva de estudiantes universitarios. Revista Iberoamericana sobre calidad, eficacia y cambio en la educación, 10 (2), 213-237.

Vera-Calzaretta, A., Carrasco-Dajer, C., da Costa, S., \& Páez, D. (2015). Factores psicosociales del presentismo en trabajadores del sistema de salud chileno. Journal of work and organizational psychology, 31, 119-128. http://dx.doi.org /10.1016/j.rpto.2015.03.004

von Sydow, M., \& Braus, N. (2016). On the tragedy of personnel evaluation. In A. Papafragou, D. Grodner, D. Mirman, \& J.C. Trueswell (Eds.), Proceedings of the ThirtyEighth Annual Conference of the Cognitive Science Society, At (Pitsburgh), Volume: 
(pp. 105-110). Austin, TX: Cognitive Science Society

Wang, J., \& Wang, X. (2012). Structural ecuation modeling. Chichester (UK): John Wiley y Sons, Ltda.

Watson, J., \& Everett, J. (1996). Small business failure rates: choice of definition and the size effect. The Journal of Entrepreneurial and Small Business Finance, 5 (3), 271-285.

West, M.A., \& Farr, J.L. (Eds.) (2009). Innovation and creativity at work: psychological and organizational strategies. Chillester (UK): John Wiley \& Sons.

Yesil, S., \& Sozbilir, F. (2013). An empirical investigation into the impact of personality on individual innovation behavior in the workplace. Social and behavioral science, 81, 540-551. http://dx.doi.org/10.1016/j.sb spro.2013.06.474

Zhao, H., Scott, S., \& Lumpkin, G. T. (2010). The relationship of personality to entrepreneurial intentions and performance: a meta-analytic review. Journal of

Management, 36 (2),

381-404. http://dx.doi.org/10.1177/014920

6309335187

\section{Notas}

* Research article. Este estudio se ha podido realizar gracias a la beca concedida a la primera autora, a la beca PSI2011-26315 y a la UFI 11/04.

1 Este autor planteaba que el mecanismo de evolución social era la supervivencia del más apto y aplicaba el concepto darwiniano de selección natural a la sociedad insistiendo en la competición étnica, nacional y de clase.

2 En la encuesta participaron al menos 1200 personas y se tienen datos parciales de 1122 .

3 Para comenzar, nos gustaría saber cómo define Ud. los conceptos de creatividad e innovación. 1. En pocas palabras, ¿qué significa para Ud. creatividad en el contexto de su organización? 2. En pocas palabras, ¿qué significa para Ud. innovación en el contexto de su organización?
4 Teniendo presente su conocimiento sobre la organización en que estudia o trabaja y los equipos, unidades, grupos de trabajo, otro de los que forma parte, le agradecemos que indique el grado en que cada afirmación es aplicable a su organización, utilizando la escala que se indica a continuación.

5 Finalmente, señale en su caso concreto 22. Considero que soy competente para el trabajo en equipo 1 = nada $234567=$ mucho.

6 Industria maquiladora

7 En el caso del factor personalidad creativa se obtuvieron solo dos y se trabajará sobre ello.

8 Y se recoge la sugerencia del método deRash como otra opción de análisis.

9 Estimado/a profesional: En su calidad de experto/a en el área de las organizaciones, calidad y/o innovación ...

10 Si bien participaron 17 jueces, uno hizo la evaluación en forma cualitativa y el resto no contestó al menos a un reactivo. Este coeficiente elimina el sujeto que no tiene todos los reactivos con valor.

11 Aunque es una dimensión sobre la que hay que seguir trabajando teniendo en cuenta indicaciones de expertos/as.

12 En las escalas setterlind a menor puntuación, más estrés.

\section{Información adicional}

Para citar este artículo: da Costa, S., Páez, D., Gondim, S., Rodríguez, M., Mazzieri, S., Torres, A., Gaudipersonas, Sánchez, F., \& Jiménez, P. (2016). Percepción de innovación en las organizaciones. Percepción de innovación en organizaciones de España y Latinoamérica. Universitas Psychologica, 15(4). http://dx.doi.org/10.11144/Javeriana.upsy154.piop 